\title{
On the Existence of Pure Strategy Nash Equilibria in Large Games*
}

\author{
Guilherme Carmona \\ Universidade Nova de Lisboa \\ Faculdade de Economia \\ Campus de Campolide, 1099-032 Lisboa, Portugal \\ email: gcarmona@fe.unl.pt \\ telephone: (351) 213801671 \\ fax: (351) 213886073
}

April 4, 2006

*I wish to thank Mário Páscoa, Myrna Wooders for very helpful comments and John Huffstot for editorial assistance. Any remaining errors are, of course, mine. 


\begin{abstract}
Over the years, several formalizations of games with a continuum of players have been given. These include those of Schmeidler (1973), Mas-Colell (1984) and Khan and Sun (1999). Unlike the others, Khan and Sun (1999) also addressed the equilibrium problem of large finite games, establishing the existence of a pure strategy approximate equilibrium in sufficiently large games. This ability for their formalization to yield asymptotic results led them to argue for it as the right approach to games with a continuum of players.

We challenge this view by establishing an equivalent asymptotic theorem based only on Mas-Colell's formalization. Furthermore, we show that it is equivalent to Mas-Colell's existence theorem. Thus, in contrast to Khan and Sun (1999), we conclude that Mas-Colell's formalization is as good as theirs for the development of the equilibrium theory of large finite games.
\end{abstract}

Keywords: Nash Equilibrium; Asymptotic Results; Pure Strategies; Approximate equilibria. 


\section{Introduction}

Nash (1950)'s celebrated existence theorem asserts that every finite normalform game has a mixed strategy equilibrium. However, in many contexts mixed strategies are unappealing and hard to interpret, leading naturally to the question of the existence of pure strategy Nash equilibria.

Schmeidler (1973) was successful in obtaining an answer to the above question. He showed that in a special class of games - in which each player's payoff depends only on his choice and on the average choice of the others a pure strategy Nash equilibrium exists in every such game with a continuum of players.

Schmeidler's formalization parallels that of Nash in that players have a finite action space, there is a function assigning to each of them a payoff function in a measurable way and the equilibrium notion is formalized in terms of a strategy, i.e., as a measurable function from players into actions. The difference is that, while in Nash (1950) there is a finite number of players (which, in particular, makes the measurability conditions trivial), in Schmeidler (1973) the set of players is the unit interval endowed with the Lebesgue measure.

Although natural, Schmeidler's formalization entails serious difficulties. As shown by Khan, Rath, and Sun (1997), Schmeidler's theorem does not extend to general games - in fact, one has to assume that either the action space or the family of payoff functions is denumerable in order to guarantee the existence of a pure strategy equilibrium (see Khan and Sun (1995) and Carmona (2005b)).

Motivated by this, Khan and Sun (1999) reformulated the theory of non- 
atomic games by modeling the set of players as a Loeb space. This reformulation allowed them to obtain an existence theorem for general games, thus dispensing with any countability assumption. This result allowed them to show that, with an appropriate assumption, all sufficiently large finite games have an approximate equilibrium. This major success, among others, led them to argue for games on Loeb spaces as the right model of large games.

In this paper, we challenge this view by proving an equivalent asymptotic theorem using neither games on Loeb spaces nor non-standard analysis (essential to their results). In fact, we show that our result, and therefore, Kahn and Sun's, is equivalent to Mas-Colell's Theorem (see Mas-Colell (1984)) on the existence of an equilibrium distribution for non-atomic games. These results suggest that Mas-Colell's formalization of the equilibrium theory of large games - in which the equilibrium notion is formulated in terms of distributions and not as a strategy - is as good as that of Khan and Sun for the development of the asymptotic theory of large games.

The equivalence between the asymptotic existence theorem and MasColell's theorem gives us a way, at least for the existence of equilibria, to go back and forth between an exact result for the non-atomic case and an approximate result for the asymptotic large finite case. We strengthen this relation between the two models by characterizing the equilibrium distributions of non-atomic games in terms of approximate equilibria in large finite games. This provides a simple and direct way of relating non-atomic games to large finite games that dispenses with the use of non-standard analysis.

Although general characterizations are possible (see Carmona (2004)), for the purpose of establishing the asymptotic existence theorem, we need 
a simple and surprisingly particular characterization result. Indeed, we can focus on games with a continuum of players with a finite action space and with finitely many characteristics belonging to an equicontinuous family. It states that a distribution of actions $\xi$ of any such game $G$ is an equilibrium if and only if for all sequences $\left\{G_{k}\right\}$ of finite games converging to $G$ (in the sense that the distributions over characteristics converge), there exists a corresponding sequence $\left\{f_{k}\right\}$ of $\varepsilon_{k}$ - equilibria with the property that $\varepsilon_{k}$ converges to 0 and the sequence of distributions induced by $f_{k}$ converges to $\xi$. It is then clear that any property that an equilibrium distribution over action has, translates into a corresponding approximate version of it for all large finite games, and vice versa. Although this characterization result holds only in the special class of games described above, we can nevertheless use it for general games, simply by approximating any such game by games in the special class in which the theorem holds. This is the reason why we can use it to establish the equivalence between the asymptotic existence theorem and Mas-Colell's theorem, which applies to general games (i.e., to games with general compact metric spaces of actions and with characteristics that may not be equicontinuous). Although the characterizations we develop in Carmona (2004) apply directly to the general case, the characterization presented here is more useful to our purpose due to the bounds on $\varepsilon_{k}$ and on the distance between the distributions induced by $f_{k}$ and $\xi$, which the special case makes possible.

The above characterization result, and those of Carmona (2004), can be interpreted as showing that Mas-Colell's model is asymptotically implementable, a criterion defended by Khan and Sun (1999), among others. Fur- 
thermore, by construction, it also frees the theory of large games from the homogeneity and measurability concerns ${ }^{1}$ by focusing directly on distributions.

As Aumann (1964) pointed out, large games are an idealization of large finite games, and so the actual space of players has no particular significance. Given this, a strategy has no more significance than the distribution it induces. As we have argued, this distribution is all we need to obtain the existence of an approximate equilibrium in large finite games. Therefore, judging Mas-Colell's distributional formalization of large games by the criteria emphasized by Khan and Sun (1999), it becomes very appealing.

The paper is organized as follows. In Section 2, we introduce our notation and basic definitions. In Section 3, we present our characterization result. Our asymptotic result and its equivalence with Mas-Colell's theorem are stated in Section 4. In Section 5, we establish several asymptotic results for games with an action space endowed with a linear structure and in which players' payoffs depend on the distribution of choices only through the average of players' choices. These results are all obtained as corollaries to our asymptotic result. In Section 6, we allow players to choose mixed strategies and players' payoffs to depend on the distribution of mixed strategies chosen. We then show that an asymptotic result also holds in this setting. In Section 7 , we provide two examples that show that neither can we obtain an exact version for our asymptotic result, nor can we dispense with the equiconti-

\footnotetext{
${ }^{1}$ The homogeneity property holds when two strategies with the same distribution can be related (by an automorphism). Regarding measurability, the concern is that requiring a strategy to be a measurable function can be a restrictive assumption, namely by imposing some sort of continuity on player's responses. See Khan and Sun (1999) for details.
} 
nuity assumption that we use. In Section 8, we present a correct version of Khan and Sun's asymptotic theorem (we give an example showing that the theorem fails as they have stated it), and then show that this correct version is equivalent to our asymptotic theorem. Section 9 concludes.

\section{Notation and Definitions}

In the class of normal-form games we consider, all players have a common pure strategy space $X$. We assume that $X$ is a compact metric space. Since the focus is on a property that depends on the number of players, we will index any game by the number of its players. Thus, $G_{n}$ is a normal-form game in which the set of players is $T_{n}=\{1, \ldots, n\}$, and each has $X$ as its choice set. A strategy is then a function $f: T_{n} \rightarrow X$. Obviously, a strategy $f$ can also be thought of as the vector $\left(f_{1}, \ldots, f_{n}\right)$ in $X^{n}$.

A game $G_{n}$ is then specified by the vector of payoff functions, one for each player. In this paper we will focus on a special class of games in which each player's payoff depends on his strategy and on the distribution of strategies chosen by the other players. Let $\mathcal{M}(X)$ be the set of Borel probability measures on $X$ endowed with the Prohorov metric $\rho$. Given a strategy $f$ in a game with $n$ players, the distribution of actions is denoted by $\nu_{n} \circ f^{-1} \in$ $\mathcal{M}(X)$ and is defined as follows:

$$
\nu_{n} \circ f^{-1}(B)=\frac{\left|\left\{t \in T_{n}: f(t) \in B\right\}\right|}{n}
$$

for any Borel set $B \subseteq X$. Clearly, $\nu_{n} \circ f^{-1}(B)$ equals the fraction of players that play an action in the set $B$. To each player $t$, we associate a continuous function $V_{n}(t): X \times \mathcal{M}(X) \rightarrow \mathbb{R}$ with the following interpretation: 
$V_{n}(t)(x, \mu)$ is player $t$ 's payoff when he plays action $x$ and facing the distribution $\mu$. Then, for any strategy $f$, player t's payoff function is

$$
U_{n}(t)(f)=V_{n}(t)\left(f(t), \nu_{n} \circ f^{-1}\right),
$$

for any strategy $f$. We denote this class of games by $\mathcal{H}$ and we represent a game $G_{n} \in \mathcal{H}$ by $G_{n}=\left(\left(T_{n}, \nu_{n}\right), V_{n}, X\right)$.

We let $\mathcal{U}$ denote the space of all continuous, real-valued functions on $X \times \mathcal{M}(X)$ with the sup norm. Thus, $V_{n}$ is a function from $T_{n}$ to $\mathcal{U}$.

Given a strategy $f, x \in X$, and $t \in T$, let $f \backslash_{t} x$ denote the strategy obtained if player $t$ changes his choice from $f(t)$ to $x$. Formally, $f \backslash_{t} x$ denotes the strategy $g$ defined by $g(t)=x$, and $g(\tilde{t})=f(\tilde{t})$, for all $\tilde{t} \neq t$. For any $\varepsilon \geq 0$, we say that $f^{*}$ is an $\varepsilon$ - equilibrium of a game $G_{n}$ if, for all $t \in T_{n}$,

$$
U_{n}(t)\left(f^{*}\right) \geq U_{n}(t)\left(f^{*} \backslash_{t} x\right)-\varepsilon \text { for all } x \in X
$$

Thus, in an $\varepsilon$ - equilibrium all players are close to their optimum by choosing according to $f^{*}$. A strategy $f^{*}$ is a Nash equilibrium of $G$ if $f^{*}$ is an $\varepsilon-$ equilibrium of $G_{n}$ for $\varepsilon=0$.

A game with a continuum of players is described by a Borel probability measure $\psi$ on $\mathcal{U}$. It can be represented by the distribution induced by a function from the unit interval, endowed with the Lebesgue measure, into $\mathcal{U}$ : $\psi=\lambda \circ V^{-1}$, where $V:[0,1] \rightarrow \mathcal{U}$ is measurable and $\lambda$ denotes the Lebesgue measure. Therefore, we also represent a game with a continuum of players by $G=(([0,1], \lambda), V, X)$.

Given a Borel probability measure $\tau$ on $\mathcal{U} \times X$, we denote by $\tau_{\mathcal{U}}$ and $\tau_{X}$ the marginal distributions of $\tau$ on $\mathcal{U}$ and $X$ respectively. The expression $u(x, \tau) \geq u(X, \tau)$ means $u(x, \tau) \geq u\left(x^{\prime}, \tau\right)$ for all $x^{\prime} \in X$. 
Given a game $\psi$ and $\varepsilon \geq 0$, a Borel probability measure $\tau$ on $\mathcal{U} \times X$ is an $\varepsilon-$ equilibrium distribution for $\psi$ if

1. $\tau_{\mathcal{U}}=\psi$, and

2. $\tau\left(\left\{(u, x) \in \mathcal{U} \times X: u\left(x, \tau_{X}\right) \geq u\left(X, \tau_{X}\right)-\varepsilon\right\}\right)=1$.

Roughly, in an $\varepsilon$ - equilibrium distribution almost all players are within $\varepsilon$ of their best replies. An equilibrium distribution is an $\varepsilon$ - equilibrium distribution with $\varepsilon=0$.

A Borel probability measure $\xi$ on $X$ is an $\varepsilon$ - equilibrium distribution over actions for $\psi$ if there exists an $\varepsilon$ - equilibrium distribution $\tau$ for $\psi$ such that $\xi=\tau_{X}$.

A strategy in a game $G=(([0,1], \lambda), V, X)$ with a continuum of players is a measurable function $f:[0,1] \rightarrow X$. For each $t \in[0,1]$, the payoff of strategy $f$ is

$$
U(t)(f)=V(t)\left(f(t), \lambda \circ f^{-1}\right) .
$$

For all $\varepsilon \geq 0$, an $\varepsilon$ - equilibrium is a strategy $f$ satisfying

$$
U(t)\left(f^{*}\right) \geq U(t)\left(f^{*} \backslash_{t} x\right)-\varepsilon
$$

for all $x \in X$ and almost all $t \in[0,1]$

Let $C$ be a finite set and $\mu$ a probability measure on $C$. In this case, we will sometimes write $\mu_{l}$ instead of $\mu(\{l\})$, whenever $l \in C$ and also $\mu=$ $\left(\mu_{1}, \ldots, \mu_{L}\right)$, with $L=|C|$. This notation also suggests that a measure with a finite support can be thought of as a vector in some Euclidean space. We will also write $\|\mu\|=\max _{l \in C}\left|\mu_{l}\right|$, i.e., $\|\mu\|$ is the sup norm of the vector 
$\left(\mu_{1}, \ldots, \mu_{L}\right)$. Note that a sequence of measures $\left\{\mu_{n}\right\}_{n=1}^{\infty}$ on $C$ converges to $\mu$ if and only if $\lim _{n \rightarrow \infty}\left\|\mu_{n}-\mu\right\|=0$.

Let $K$ be a subset of $\mathcal{U}$. We say that $K$ is equicontinuous (or, that the family $K$ of functions is equicontinuous) if for all $\eta>0$ there exists a $\delta>0$ such that $\max \{\rho(\mu, \tau), d(x, y)\}<\delta$ implies

$$
|V(x, \mu)-V(y, \tau)|<\eta
$$

for all $V \in K$ and for all $x, y \in X$ and $\mu, \tau \in \mathcal{M}(X)$ (see Rudin (1976, p. 156)). In our framework, equicontinuity can be interpreted as placing "a bound on the diversity of payoffs" (see Khan, Rath, and Sun (1997)). For all $\delta>0$, let

$$
\omega_{V}(\delta)=\sup \{|V(x, \mu)-V(y, \tau)|: \max \{d(x, y), \rho(\mu, \tau)\}<\delta\}
$$

denote the modulus of continuity of $V$ and

$$
\omega_{K}(\delta)=\sup _{V \in K} \omega_{V}(\delta)
$$

Of course, if $K$ is equicontinuous, then $\lim _{\delta \rightarrow 0} \omega_{K}(\delta)=0$.

\section{A Characterization of Equilibrium Distri- butions}

In this section we characterize equilibrium distributions of some simple games with a continuum of players. These are games with a finite number of characteristics and actions and with payoff functions selected from an equicontinuous family. Despite all these restrictive assumptions, this result is enough to 
establish the existence of pure strategy approximate equilibria in large finite games.

Theorem 1 Let $X$ be a finite set, $m=|X|$ and $K$ be an equicontinuous subset of $\mathcal{U}$. Then, the following holds for all games $G=(([0,1], \lambda), V, X)$ with a continuum of players such that $V([0,1])$ is a finite subset of $K$ and for all $\varepsilon \geq 0$ :

$A$ distribution $\xi$ on $X$ is an $\varepsilon$ - equilibrium distribution over actions of $G$ if and only if for all games $G_{n}=\left(\left(T_{n}, \nu_{n}\right), V_{n}, X\right)$ with a finite number of players in which $V_{n}\left(T_{n}\right)$ is a subset of $V([0,1])$ there exists a strategy $f_{n}: T_{n} \rightarrow X$ such that

1. $f_{n}$ is $\varepsilon+2 \omega_{K}\left(m\left\|\lambda \circ V^{-1}-\nu_{n} \circ V_{n}^{-1}\right\|+\left(m^{2}+1\right) / n\right)-$ equilibrium of $G_{n}$ and

2. $\left\|\nu_{n} \circ f_{n}^{-1}-\xi\right\| \leq\left\|\lambda \circ V^{-1}-\nu_{n} \circ V_{n}^{-1}\right\|+\frac{m}{n}$.

In order to illustrate the idea of Theorem 1, consider the particular case of a sequence of games $\left\{G_{n}\right\}$ with a finite number of players with $V_{n}\left(T_{n}\right) \subseteq$ $V([0,1])$ and with $\left\|\lambda \circ V^{-1}-\nu_{n} \circ V_{n}^{-1}\right\|$ converging to zero. In this case, we can, intuitively, say that the sequence $\left\{G_{n}\right\}$ converges to $G$. If $\xi$ is an equilibrium distribution over actions of $G$, then Theorem 1 guarantees the existence of an $\varepsilon_{n}-$ equilibrium $f_{n}$ of $G_{n}$ satisfying $\varepsilon_{n} \rightarrow 0$ and $\left\|\nu_{n} \circ f_{n}^{-1}-\xi\right\| \rightarrow 0$. That is, finite games that are close to $G$ have approximate equilibria, with a degree of approximation close to zero, whose induced distributions are close to $\xi$.

Conversely, the existence of approximate equilibria of games converging to $G$, with a vanishing degree of approximation and with induced distributions 
converging to $\xi$, is enough to show that $\xi$ is an equilibrium distribution over actions of $G$.

The strength of Theorem 1, which is crucial to the asymptotic result, is that the degree of approximation involved depends only on $\varepsilon$, on the equicontinuous family $K$, on the number of pure strategies $m$, on the Euclidian distance between the distributions of characteristics $\left\|\lambda \circ V^{-1}-\nu_{n} \circ V_{n}^{-1}\right\|$ and on the number of players $n$. In particular, it is independent of the particular games $G$ and $G_{n}$ that we are considering. So, if $\varepsilon$ and the set of actions is fixed, and we are considering games $G$ and $G_{n}$ with the same distribution of characteristics, then the degree of approximation depends only on $n$. This fact is at the core of our asymptotic result: once $n$ is sufficiently large, equilibria will have nice properties.

Proof of Theorem 1. Let $X$ be a finite set and $K$ be an equicontinuous subset of $\mathcal{U}$. Let $\varepsilon \geq 0$ and let $G=(([0,1], \lambda), V, X)$ be a game with a continuum of players such that $V([0,1])$ is a finite subset of $K$. Let $\beta=$ $\lambda \circ V^{-1}$. Let $\operatorname{supp}(\beta)=\left\{V_{1}, \ldots, V_{L}\right\}$.

(Necessity) Let $\xi$ be an $\varepsilon$ - equilibrium distribution over actions of $G$ and let $\mu$ be an $\varepsilon-$ equilibrium distribution of $G$ such that $\xi=\mu_{X}$. We can represent $\mu$ as follows:

\begin{tabular}{cccc}
$\mu_{1,1}$ & $\mu_{1,2}$ & $\cdots$ & $\mu_{1, m}$ \\
$\mu_{2,1}$ & $\mu_{2,2}$ & & $\mu_{2, m}$ \\
$\vdots$ & & & \\
$\mu_{L, 1}$ & $\mu_{L, 2}$ & & $\mu_{L, m}$ \\
\hline$\xi_{1}$ & $\xi_{2}$ & & $\xi_{m}$
\end{tabular}


Note that $\sum_{i=1}^{m} \mu_{l, i}=\beta_{l}$ for all $1 \leq l \leq L$. Since $\mu$ is an $\varepsilon-$ equilibrium distribution, it follows that if $\mu_{l, i}>0$ then

$$
V_{l}\left(x_{i}, \xi\right) \geq V_{l}(x, \xi)-\varepsilon
$$

for all $x \in X$.

Let $G_{n}$ be a game with a finite number of players such that $V_{n}\left(T_{n}\right)$ is a subset of $V([0,1])$. For all $1 \leq l \leq L$, let $T_{n, l}=\left\{t \in T_{n}: V_{n}(t)=V_{l}\right\}$ and $\gamma_{n, l}=\left|T_{n, l}\right|$. Then, $\gamma_{n}=\left(\gamma_{n, 1}, \ldots, \gamma_{n, L}\right)$ is such that $\gamma_{n} / n=\nu_{n} \circ V_{n}^{-1}$.

Let $1 \leq l \leq L$ be given. Define

$$
S_{l}=\left\{e_{i}: \mu_{l, i}>0\right\},
$$

where $E=\left\{e_{1}, \ldots, e_{m}\right\}$ is the standard basis of $\mathbb{R}^{m}$. Define $S_{t}=S_{l}$ if $t \in T_{n, l}$.

If $\gamma_{n, l}>0$, it follows that, $S_{l} \subseteq \frac{1}{\gamma_{n, l}} \sum_{t \in T_{n, l}} S_{t}$. Also, we have that

$$
\frac{\mu_{l}}{\beta_{l}}=\left(\frac{\mu_{l, 1}}{\beta_{l}}, \ldots, \frac{\mu_{l, m}}{\beta_{l}}\right) \in \operatorname{co}\left(S_{l}\right)
$$

and so

$$
\frac{\mu_{l}}{\beta_{l}} \in \operatorname{co}\left(\frac{1}{\gamma_{n, l}} \sum_{t \in T_{n, l}} S_{t}\right)=\frac{1}{\gamma_{n, l}} \sum_{t \in T_{n, l}} \operatorname{co}\left(S_{t}\right) .
$$

Define

$$
\tau=\sum_{l=1}^{L} \frac{\gamma_{n, l}}{n} \frac{\mu_{l}}{\beta_{l}}=\sum_{l: \gamma_{n, l}>0} \frac{\gamma_{n, l}}{n} \frac{\mu_{l}}{\beta_{l}} .
$$

Then, for all $1 \leq i \leq m$, it follows that

$$
\left|\mu_{i}-\tau_{i}\right| \leq \sum_{l=1}^{L} \frac{\mu_{l, i}}{\beta_{l}}\left|\frac{\gamma_{n, l}}{n}-\beta_{l}\right| \leq\left\|\beta-\frac{\gamma_{n}}{n}\right\|,
$$

and so

$$
\|\xi-\tau\| \leq\left\|\beta-\frac{\gamma_{n}}{n}\right\|
$$


Hence, by Lemma 1,

$$
\rho(\xi, \tau) \leq m\left\|\beta-\frac{\gamma_{n}}{n}\right\|
$$

Furthermore,

$$
\tau \in \sum_{l: \gamma_{n, l}>0} \frac{\gamma_{n, l}}{n} \frac{1}{\gamma_{n, l}} \sum_{t \in T_{n, l}} \operatorname{co}\left(S_{t}\right)=\frac{1}{n} \sum_{t \in T_{n}} \operatorname{co}\left(S_{t}\right) .
$$

Thus, by the Shapley-Folkman Theorem (see Rashid (1983, p. 9)), it follows that there are $n$ points $\left(\alpha_{t}\right)_{t \in T_{n}}$ such that $\alpha_{t} \in \operatorname{co}\left(S_{t}\right)$, for all $t \in T_{n}$,

$$
\tau=\frac{1}{n} \sum_{t \in T_{n}} \alpha_{t}
$$

and

$$
\left|\left\{t \in T_{n}: \alpha_{t} \notin S_{t}\right\}\right| \leq m
$$

Let $1 \leq l \leq L$ and define $P_{n}=\left\{t \in T_{n}: \alpha_{t} \in E\right\}$. Define a strategy $f_{n}$ as follows: if $t \in P_{n}$, then let $e_{i}$ be such that $\alpha_{t}=e_{i}$ and define $f_{n}(t)=x_{i}$; if $t \in P_{n}^{c}:=T_{n} \backslash P_{n}$ and $V_{t}=V_{l}$, choose $1 \leq i \leq m$ such that $\mu_{l, i}>0$ and define $f_{n}(t)=x_{i}$. By $(6)$, it follows that

$$
V_{t}\left(f_{n}(t), \xi\right) \geq V_{t}(x, \xi)-\varepsilon
$$

for all $t \in T_{n}$ and $x \in X$.

Let $\sigma=\nu_{n} \circ f_{n}^{-1}$. We claim that

$$
\|\tau-\sigma\| \leq \frac{m}{n}
$$

Let $1 \leq i \leq m$. We have that

$$
\sigma_{i}=\sum_{t \in P_{n}} \frac{\alpha_{t}(i)}{n}+\sum_{t \in P_{n}^{c} \cap f_{n}^{-1}\left(x_{i}\right)} \frac{1}{n}
$$


and

$$
\tau_{i}=\sum_{t=1}^{n} \frac{\alpha_{t}(i)}{n}
$$

Therefore, letting $\chi_{f^{-1}\left(x_{i}\right)}$ denote the characteristic function of $f^{-1}\left(x_{i}\right)$, we obtain that

$$
\left|\tau_{i}-\sigma_{i}\right|=\frac{1}{n}\left|\sum_{t \in P_{n}^{c}}\left(\alpha_{t}(i)-\chi_{f^{-1}\left(x_{i}\right)}\right)\right| \leq \frac{1}{n} \sum_{t \in P_{n}^{c}}\left|\alpha_{t}(i)-\chi_{f^{-1}\left(x_{i}\right)}\right| \leq \frac{m}{n}
$$

and so $\|\tau-\sigma\| \leq m / n$.

Since $\nu_{n} \circ f^{-1}=\sigma$ and $\|\xi-\tau\| \leq\left\|\beta-\gamma_{n} / n\right\|$, then $\left\|\nu_{n} \circ f^{-1}-\xi\right\| \leq$ $\left\|\beta-\gamma_{n} / n\right\|+m / n$. This establishes assertion 2 in the statement of the Theorem.

By Lemma $1, \rho\left(\tau, \nu_{n} \circ f_{n}^{-1}\right) \leq m^{2} / n$ since $\nu_{n} \circ f_{n}^{-1}=\sigma$. Also, by Lemma 2 , it follows that

$$
\rho\left(\nu_{n} \circ f_{n}^{-1}, \nu_{n} \circ\left(f_{n} \backslash_{t} x\right)^{-1}\right) \leq \frac{1}{n}
$$

for all $t \in T_{n}$ and $x \in X$. Hence, using (9), it follows that

$$
\rho\left(\nu_{n} \circ f_{n}^{-1}, \xi\right) \leq m\left\|\beta-\frac{\gamma_{n}}{n}\right\|+\frac{m^{2}}{n}
$$

and

$$
\rho\left(\nu_{n} \circ\left(f_{n} \backslash_{t} x\right)^{-1}, \xi\right) \leq m\left\|\beta-\frac{\gamma_{n}}{n}\right\|+\frac{m^{2}+1}{n} .
$$

For convenience, let $\theta=m\left\|\beta-\frac{\gamma_{n}}{n}\right\|+\frac{m^{2}+1}{n}$. Hence, for all $t \in T_{n}$ and $x \in X$, we obtain

$$
\begin{aligned}
V_{t}\left(f_{n}(t), \nu_{n} \circ f_{n}^{-1}\right) & \geq V_{t}\left(f_{n}(t), \xi\right)-\omega_{K}(\theta) \\
& \geq V_{t}(x, \xi)-\varepsilon-\omega_{K}(\theta) \\
& \geq V_{t}\left(x, \nu_{n} \circ\left(f_{n} \backslash_{t} x\right)^{-1}\right)-\varepsilon-2 \omega_{K}(\theta) .
\end{aligned}
$$


Therefore, $f_{n}$ is a pure $\varepsilon+2 \omega_{K}\left(m\left\|\beta-\gamma_{n} / n\right\|+\left(m^{2}+1\right) / n\right)-$ equilibrium of $G_{n}$.

(Sufficiency) Let $\xi$ be a distribution over $X$ satisfying the condition. Let $\left\{q_{n}\right\} \subseteq \mathbb{Q}_{+}^{L}$ be such that $q_{n} \rightarrow \beta$. Consider first the case in which there exists $\gamma_{n}=\left(\gamma_{n, 1}, \ldots, \gamma_{n, L}\right) \in \mathbb{N}^{L}$ such that $q_{n}=\gamma_{n} / n$, for all $n \in \mathbb{N}$. Define, for all $n$, a game $G_{n}=\left(\left(T_{n}, \nu_{n}\right), V_{n}, X\right)$ where $V_{n}$ satisfies $\left|\left\{t \in T_{n}: V_{n}(t)=V_{l}\right\}\right|=$ $\gamma_{n, l}$ for all $1 \leq l \leq L$.

For all $n$, let $f_{n}$ satisfy 1 and 2 . Consider the sequence $\left\{\nu_{n} \circ\left(V_{n}, f_{n}\right)^{-1}\right\}$. Taking a subsequence if necessary, we may assume that it converges. Let $\mu=\lim _{n} \nu_{n} \circ\left(V_{n}, f_{n}\right)^{-1}$. Then, $\mu_{\mathcal{U}}=\beta=\lambda \circ V^{-1}$ and $\mu_{X}=\xi$ since, respectively, $\nu_{n} \circ V_{n}^{-1}=\gamma_{n} / n$ and $\left\|\nu_{n} \circ f_{n}^{-1}-\xi\right\| \leq\left\|\beta-\gamma_{n} / n\right\|+m / n \rightarrow 0$. Since $\nu_{n} \circ\left(V_{n}, f_{n}\right)^{-1}$ converges to $\mu, f_{n}$ is an $\varepsilon+2 \omega_{K}\left(m\left\|\beta-\frac{\gamma_{n}}{n}\right\|+\frac{m^{2}+1}{n}\right)-$ equilibrium of $G_{n}$ and $\lim _{n}\left(m\left\|\beta-\frac{\gamma_{n}}{n}\right\|+\frac{m^{2}+1}{n}\right)=0$, it follows, by Lemma 5 , that $\mu$ is an $\varepsilon$ - equilibrium distribution of $G$. Thus, $\xi$ is an $\varepsilon$ - equilibrium distribution over action of $G$.

We turn now to the general case. For all $n \in \mathbb{N}$, there exists $\alpha_{n} \in \mathbb{N}^{L}$ and $m_{n} \in \mathbb{N}$ such that $q_{n}=\alpha_{n} / m_{n}$ and $\left\{m_{n}\right\}_{n}$ is increasing. Consider a sequence $\left\{\sigma_{k} / k\right\}$ satisfying $\sigma_{k}=\alpha_{n}$ if $k=m_{n}$. Defining a game $G_{k}$ as above, we obtain a strategy $f_{k}$ satisfying 1 and 2 , for all $k \in \mathbb{N}$. Then, we consider the subsequence $\left\{\nu_{k_{j}} \circ\left(V_{k_{j}}, f_{k_{j}}\right)^{-1}\right\}_{j}$, where $k_{j}=m_{j}$ for all $j \in \mathbb{N}$. Then, $\nu_{k_{j}} \circ V_{k_{j}}^{-1}$ converges to $\beta$, and we can use a similar argument as above. 


\section{Existence of Pure, Approximate Equilibria in Large, Equicontinuous Games}

In this section we state our asymptotic result. It says that all sufficiently large games have a pure $\varepsilon$ - equilibrium, provided that players' payoff functions are selected from an equicontinuous family.

Theorem 2 Let $K$ be an equicontinuous subset of $\mathcal{U}$. Then, for all $\varepsilon>0$ there exists $N \in \mathbb{N}$ such that $n \geq N$ and $V_{n}\left(T_{n}\right) \subseteq K$ implies that $G_{n} \in \mathcal{H}$ has a pure $\varepsilon$ - equilibrium.

Theorem 2 is an approximation result: it guarantees the existence of an approximate equilibrium in pure strategies if the game is sufficiently large. Essentially, we are approximating not only pure strategy Nash equilibria, but also games with a continuum of players, in which the conclusion of Theorem 2 holds exactly. This is how we proceed: we associate, to any sufficiently large finite game, a game with a continuum of players having the same distribution of payoff functions. By Mas-Colell's existence theorem, we obtain an equilibrium distribution. An important lemma (Lemma 4 in Appendix A.1) then shows that there exists an approximate equilibrium with finite support. We then restrict players to this finite set of actions, and since the set of players characteristic is a finite subset of an equicontinuous family, we can apply Theorem 1 to this approximate equilibrium. In this way, we construct a pure approximate equilibrium for the original finite game.

Proof of Theorem 2. Let $\varepsilon>0$. Let $\delta>0$ be such that $\omega_{K}(\delta)<\varepsilon / 3$ and let $\left\{x_{1}, \ldots, x_{m}\right\} \subseteq X$ be given by Lemma 4 and corresponding to $\eta=$ 
$\min \{\varepsilon / 3, \delta\}$. Finally, let $N \in \mathbb{N}$ be such that $\omega_{K}\left(\frac{m^{2}+1}{n}\right)<\varepsilon / 3$ and $1 / n<\delta$ for all $n \geq N$.

Let $G_{n}$ be a game in $\mathcal{H}$ with $n \geq N$. Consider the following game with a continuum of players: $G=(([0,1], \lambda), V, X)$ where $V(t)=V_{n}(i)$ if $t \in T_{i}:=\left[\frac{i-1}{n}, \frac{i}{n}\right)$ for $1 \leq i \leq n-1$ and $V(t)=V_{n}(n)$ if $t \in T_{n}=\left[\frac{n-1}{n}, 1\right]$. Note that $\lambda \circ V^{-1}=\nu_{n} \circ V_{n}^{-1}$. Since $V([0,1])$ is finite and is a subset of $K$, it follows by Lemma 4 that $G$ has $\varepsilon / 3$ - equilibrium $f$ with $f([0,1]) \subseteq\left\{x_{1}, \ldots, x_{m}\right\}$.

By Theorem 1 , there exists a $\varepsilon / 3+\omega_{K}\left(\left(m^{2}+1\right) / n\right)$ - equilibrium $f_{n}$ of the game $\left(\left(T_{n}, \nu_{n}\right), V_{n},\left\{x_{1}, \ldots, x_{m}\right\}\right)$. Let $t \in T_{n}$ and $x \in X$. Then, there exists $x_{i} \in\left\{x_{1}, \ldots, x_{m}\right\}$ such that $d\left(x, x_{i}\right)<\delta$. Note that $\rho\left(\nu_{n} \circ\left(f_{n} \backslash_{t} x_{i}\right)^{-1}, \nu_{n} \circ\right.$ $\left.\left(f_{n} \backslash_{t} x\right)^{-1}\right)<1 / n<\delta$. Hence,

$$
\begin{aligned}
V_{t}\left(f_{n}(t), \nu_{n} \circ f_{n}^{-1}\right) & \geq V_{t}\left(x_{i}, \nu_{n} \circ\left(f_{n} \backslash_{t} x_{i}\right)^{-1}\right)-\left(\frac{\varepsilon}{3}+\omega_{K}\left(\frac{m^{2}+1}{n}\right)\right) \\
& \geq V_{t}\left(x, \nu_{n} \circ\left(f_{n} \backslash_{t} x\right)^{-1}\right)-\left(\frac{\varepsilon}{3}+\omega_{K}\left(\frac{m^{2}+1}{n}\right)+\omega_{K}(\delta)\right)(15 \\
& >V_{t}\left(x, \nu_{n} \circ\left(f_{n} \backslash_{t} x\right)^{-1}\right)-\varepsilon .
\end{aligned}
$$

Therefore, $f_{n}$ is an $\varepsilon$ - equilibrium of $G_{n}$.

Theorem 2 is clearly related to Mas-Colell's, which states that an equilibrium exists for all games with a continuum of players.

Theorem 3 (Mas-Colell) An equilibrium distribution exists for all games $\mu \in \mathcal{M}(\mathcal{U})$.

In fact, our construction shows that Theorem 2 is a consequence of MasColell's existence theorem. But more is true: as Theorem 4 below shows, the two results are equivalent. This implies that Theorem 2 is the asymptotic version of Mas-Colell's existence theorem. 
Theorem 4 Theorem 2 holds if and only if Theorem 3 holds.

The equivalence between the two results means that they are simply two different ways of expressing the same phenomenon: the existence of pure strategy Nash equilibria can be addressed either in its exact version in games with a continuum of players or in an approximate version in large, equicontinuous games. This result clearly stresses the relation between equilibrium distributions of games with a continuum of players and approximate equilibria of large finite games.

Proof of Theorem 4. Since we have established Theorem 2 using Theorem 3 (which is essential to Lemma 4), it is enough to show that we can prove Theorem 3 with Theorem 2.

Let $\mu$ be a game with a continuum of players. Then, by Parthasarathy (1967, Theorem II.6.3, p.44), there exists a sequence $\left\{\mu_{k}\right\} \subseteq \mathcal{M}(\mathcal{U})$ such that $\mu_{k}$ converges to $\mu, \operatorname{supp}\left(\mu_{k}\right)$ is finite and $\mu_{k}(\{v\}) \in \mathbb{Q}$ for all $v \in \operatorname{supp}\left(\mu_{k}\right)$. Let $\operatorname{supp}\left(\mu_{k}\right)=\left\{V_{k}^{1}, \ldots, V_{k}^{L_{k}}\right\}$, where $V_{k}^{l} \in \mathcal{U}$ for all $1 \leq l \leq L_{k}$ and

$$
\frac{\beta_{k}^{l}}{t_{k}}=\mu_{k}\left(\left\{V_{k}^{l}\right\}\right)
$$

Let $k \in \mathbb{N}$ be fixed. Then $\left\{V_{k}^{l}\right\}_{1 \leq l \leq L_{k}}$ is an equicontinuous subset of $\mathcal{U}$. Define the following set of games $G_{\gamma t_{k}}=\left(\left(T_{\gamma t_{k}}, \nu_{\gamma t_{k}}\right), V_{\gamma t_{k}}, X\right)$ for all $\gamma \in \mathbb{N}$. That is, $G_{\gamma t_{k}}$ has $\gamma t_{k}$ players, each has $X$ as his choice set and their payoff functions are defined in the following way: $V_{\gamma t_{k}}: T_{\gamma t_{k}} \rightarrow \mathcal{U}$ is such that it associates $V_{k}^{l}$ to $\gamma \beta_{k}^{l}$ players, for all $1 \leq l \leq L_{k}$.

By Theorem 2, $G_{\gamma_{k} t_{k}}$ has a $1 / k$ - equilibrium $f_{\gamma_{k} t_{k}}: T_{\gamma_{k} t_{k}} \rightarrow X$ if $\gamma_{k}$ is sufficiently large. Let $\tau_{k}=\nu_{\gamma_{k} t_{k}} \circ\left(V_{\gamma_{k} t_{k}}, f_{\gamma_{k} t_{k}}\right)^{-1} \in \mathcal{M}(\mathcal{U} \times X)$. We may assume that $\gamma_{k} t_{k}>k$, by choosing $\gamma_{k}$ large enough. 
Since $\tau_{\mathcal{U}, k}$ converges to $\mu$, it follows that $\left\{\mu, \tau_{\mathcal{U}, 1}, \tau_{\mathcal{U}, 2}, \ldots\right\}$, and so $\left\{\tau_{\mathcal{U}, k}\right\}_{k}$ is tight by Hildenbrand (1974, Theorem 32 and 33, p. 49 and 50). Also, since $\mathcal{M}(X)$ is compact, then $\left\{\tau_{X, 1}, \tau_{X, 2}, \ldots\right\}$ is tight by Hildenbrand (1974, Theorem 34, p. 50). Thus, $\left\{\tau_{k}\right\}_{k}$ is tight (Hildenbrand (1974, Theorem 35, p. 50)) and, taking a subsequence if necessary, we may assume that $\left\{\tau_{k}\right\}$ converges (Hildenbrand (1974, Theorem 31, p. 49)). Let $\tau=\lim _{k} \tau_{k}$. Then, by Lemma 5 it follows that $\tau$ is an equilibrium distribution of $\tau_{\mathcal{U}}=\mu$.

Another aspect that stresses the asymptotic nature of Theorem 2 is that it requires a class of payoff functions for which an equilibrium is sure to exist whenever the game has a continuum of players. In particular, it is false for games in which players have general payoff functions. As a simple example, consider $\varepsilon=1 / 4$, and the following games $G_{n}$ with $X=\{H, T\}$ and $n \geq 2$ : players 1 and 2 play the matching pennies (see Table 1 ), while the remaining players are indifferent between all strategies (i.e., $U_{n}(t)(f)=0$ for all strategies $f$ ).

\begin{tabular}{|c|c|c|}
\hline $1 \backslash 2$ & $H$ & $T$ \\
\hline$H$ & $1,-1$ & $-1,1$ \\
\hline$T$ & $-1,1$ & $1,-1$ \\
\hline
\end{tabular}

Table 1: Payoff Function for the Matching Pennies

Then, if $f$ is a pure strategy, it follows that at least one of players 1 and 2 is not $1 / 4$ - optimizing. This implies that $f$ cannot be a $1 / 4$ - equilibrium. Therefore, $G_{n}$ has no pure, $1 / 4$ - equilibrium for all $n \geq 2$. 


\section{Games based on the Averages of Individual Choices}

Khan and Sun (1999) also consider the case in which the action space is endowed with a linear structure and each player's payoff depends only on his choice and on the average of players' choices. In this section, we establish an existence result for this case, as well. In particular, we show that an approximate equilibrium exists in all equicontinuous, and sufficiently large games if

1. the action space is a weakly compact subset of a separable Banach space and the average is a Bochner integral,

2. the action space is a norm compact subset of a Banach space and the average is again a Bochner integral,

3. the action space is a weak* compact subset of the dual of a separable Banach space and the average is a Gel'fand integral and

4. the action space is a denumerable subset of $\mathbb{R}^{\infty}$.

We emphasize that these results are obtained as corollaries of Theorem 2. Although a direct argument is possible, this highlights the additional generality of considering games in which each player's payoff depends on the distribution of players' choices when compared with games in which it depends only on the average of players' choices. 


\subsection{Bochner Integral with the Weak Topology}

In this subsection, assume that $X$ is a weakly compact subset of a separable Banach space $B$. Let $\overline{\mathrm{co}}(X)$ denote the closed convex hull of $X$, which is also weakly compact (see Diestel and Uhl (1977, Theorem 11, p. 51)). It follows from Dunford and Schwartz (1957, Theorem V.6.3, p. 434) that both $X$ and $\overline{\mathrm{co}}(X)$ are metrizable. Finally, let $\mathcal{U}_{w}$ be the space of weakly continuous real-valued functions on $X \times \overline{\mathrm{co}}(X)$ endowed with the sup norm.

If $f: T_{n} \rightarrow X$ is a pure strategy in a game with $n$ players, let $\int_{T_{n}} f \mathrm{~d} \nu_{n}$ denote its Bochner integral. Clearly,

$$
\int_{T_{n}} f \mathrm{~d} \nu_{n}=\frac{1}{n} \sum_{t=1}^{n} f(t) \in \overline{\mathrm{co}}(X) .
$$

In this case, we associate to each player $t$ a weakly continuous function $V_{n}(t)$ : $X \times \overline{\mathrm{co}}(X) \rightarrow \mathbb{R}$ and define player $t$ 's payoff function as

$$
U_{n}(t)(f)=V_{n}(t)\left(f(t), \int_{T_{n}} f \mathrm{~d} \nu_{n}\right),
$$

for any strategy $f: T_{n} \rightarrow X$. We denote this class of games by $\mathcal{H}_{w}$.

Corollary 1 Let $K$ be an equicontinuous subset of $\mathcal{U}_{w}$. Then, for all $\varepsilon>0$ there exists $N \in \mathbb{N}$ such that $n \geq N$ and $V_{n}\left(T_{n}\right) \subseteq K$ implies that $G_{n} \in \mathcal{H}_{w}$ has a pure $\varepsilon$ - equilibrium.

\subsection{Bochner Integral with the Norm Topology}

In this subsection, assume that $X$ is a norm compact of a Banach space $B$. Then, $\overline{\mathrm{co}}(X)$ is also norm compact (see Diestel and Uhl (1977, Theorem 12, p. 51)). We let $\mathcal{U}_{\text {norm }}$ be the space of norm continuous real-valued functions on $X \times \overline{\mathrm{co}}(X)$ endowed with the sup norm. 
Finally, we let $\mathcal{H}_{\text {norm }}$ be the class of games defined as in Subsection 5.1, except that now players' payoff functions belong to $\mathcal{U}_{\text {norm }}$ (and $X$ is norm compact).

As in Khan, Rath, and Sun (1997), Corollary 1 also applies to the case of norm compact action spaces. Note that $X$ is weakly compact. Thus, since norm continuous functions on norm compact sets are weakly continuous, we obtain the following existence result.

Corollary 2 Let $K$ be an equicontinuous subset of $\mathcal{U}_{\text {norm. }}$. Then, for all $\varepsilon>0$ there exists $N \in \mathbb{N}$ such that $n \geq N$ and $V_{n}\left(T_{n}\right) \subseteq K$ implies that $G_{n} \in \mathcal{H}_{\text {norm }}$ has a pure $\varepsilon$ - equilibrium.

\subsection{Gel'fand Integral}

In this subsection, let $B^{*}$ be the dual of a separable Banach space $B$ and assume that $X$ is a weak* compact subset of $B^{*}$. Then, $\overline{\mathrm{co}}(X)$ is also weak* compact. It follows from Rudin (1971, Theorem 3.16, p. 70) that both $X$ and $\overline{\mathrm{co}}(X)$ are metrizable. Finally, let $\mathcal{U}_{g}$ be the space of weak* continuous real-valued functions on $X \times \overline{\mathrm{co}}(X)$ endowed with the sup norm.

If $f: T_{n} \rightarrow X$ is a pure strategy in a game with $n$ players, let $\int_{T_{n}} f \mathrm{~d} \nu_{n}$ denote its Gel'fand integral. Clearly,

$$
\int_{T_{n}} f \mathrm{~d} \nu_{n}=\frac{1}{n} \sum_{t=1}^{n} f(t) \in \overline{\mathrm{co}}(X) .
$$

We associate to each player $t$ a weak ${ }^{*}$ continuous function $V_{n}(t): X \times$ $\overline{\mathrm{co}}(X) \rightarrow \mathbb{R}$ and define player $t$ 's payoff function as

$$
U_{n}(t)(f)=V_{n}(t)\left(f(t), \int_{T_{n}} f \mathrm{~d} \nu_{n}\right)
$$


for any strategy $f: T_{n} \rightarrow X$. We denote this class of games by $\mathcal{H}_{g}$.

Corollary 3 Let $K$ be an equicontinuous subset of $\mathcal{U}_{g}$. Then, for all $\varepsilon>0$ there exists $N \in \mathbb{N}$ such that $n \geq N$ and $V_{n}\left(T_{n}\right) \subseteq K$ implies that $G_{n} \in \mathcal{H}_{g}$ has a pure $\varepsilon$ - equilibrium.

\subsection{Games with Denumerably Many Actions in $\mathbb{R}^{\infty}$}

In this subsection, we consider the class of games defined in Section 5 of Khan, Rath, and Sun (1997). Let $\mathbb{R}^{\infty}$ be the space of all real sequences equipped with the product topology. The standard basis vectors are denoted by $\left\{e_{i}\right\}_{i=1}^{\infty}$. We let the space of actions be $X=\left\{e_{i}\right\}_{i=0}^{\infty}$ with $e_{0} \equiv 0$. Again, $\overline{\mathrm{co}}(X)$ denotes the closed convex hull of $X$, which is equal to the set of all sequences of real numbers chosen from the closed unit interval. Finally, let $\mathcal{U}_{\infty}$ be the space of continuous real-valued functions on $X \times \overline{\mathrm{co}}(X)$ endowed with the sup norm.

The following definitions are from Khan, Rath, and Sun (1997). If $(T, \mathcal{T}, \nu)$ is a probability space, then a function $f: T \rightarrow \mathbb{R}^{\infty}$ is measurable if $f^{-1}\left(\left\{e_{i}\right\}\right) \in$ $\mathcal{T}$. In this case,

$$
\int_{T} f \mathrm{~d} \nu=\sum_{i=1}^{\infty} e_{i} \nu\left(f^{-1}\left(\left\{e_{i}\right\}\right)\right) .
$$

In particular, if $\mu \in \mathcal{M}(X)$, then $\int_{X} i \mathrm{~d} \mu=\sum_{i=1}^{\infty} e_{i} \mu\left(\left\{e_{i}\right\}\right)$ and so we trivially obtain the following change of variable formula:

$$
\int_{T} f \mathrm{~d} \nu=\int_{X} i \mathrm{~d} \nu \circ f^{-1}
$$

Furthermore, if $j \in \mathbb{N}$ and $\pi_{j}: \mathbb{R}^{\infty} \rightarrow \mathbb{R}$ is the projection onto the $j^{\text {th }}$ coordinate, then $\pi_{j}$ is a simple function and its Lebesgue integral is $\int_{X} \pi_{j} \mathrm{~d} \mu=$ $\mu\left(\left\{e_{j}\right\}\right)$. 
If $f: T_{n} \rightarrow X$ is a pure strategy in a game with $n$ players, then, clearly,

$$
\int_{T_{n}} f \mathrm{~d} \nu_{n}=\frac{1}{n} \sum_{t=1}^{n} f(t) \in \overline{\mathrm{co}}(X) .
$$

In this case, we associate to each player $t$ a continuous function $V_{n}(t)$ : $X \times \overline{\mathrm{co}}(X) \rightarrow \mathbb{R}$ and define player $t$ 's payoff function as

$$
U_{n}(t)(f)=V_{n}(t)\left(f(t), \int_{T_{n}} f \mathrm{~d} \nu_{n}\right),
$$

for any strategy $f$. We denote this class of games by $\mathcal{H}_{\infty}$.

Corollary 4 Let $K$ be an equicontinuous subset of $\mathcal{U}_{\infty}$. Then, for all $\varepsilon>0$ there exists $N \in \mathbb{N}$ such that $n \geq N$ and $V_{n}\left(T_{n}\right) \subseteq K$ implies that $G_{n} \in \mathcal{H}_{\infty}$ has a pure $\varepsilon$ - equilibrium.

\section{$6 \quad$ Mixed Strategies}

In this section, we allow players to choose mixed strategies. In this context, a mixed strategy for a player is a Borel probability measure on the set of his pure strategies. Thus, a mixed strategy is a function $f: T_{n} \rightarrow \mathcal{M}(X)$, while a pure strategy is a function $g: T_{n} \rightarrow X$. Note that a pure strategy can be seen as a mixed strategy, simply by associating $X$ with the degenerate probability measures on $X$.

Given a strategy $f$ in a game with $n$ players, the distribution of players' choices is $\nu_{n} \circ f^{-1}$, now an element of $\mathcal{M}(\mathcal{M}(X))$. Consequently, players' payoff functions are elements of $\mathcal{U}_{m}$, which we use to denote the space of all continuous, real valued functions on $X \times \mathcal{M}(\mathcal{M}(X))$. 
As in Section 2, we associate to each player a function $V_{n}(t): X \times$ $\mathcal{M}(\mathcal{M}(X)) \rightarrow \mathbb{R}$ with the following interpretation: $V_{n}(t)(x, \mu)$ is player $t$ 's payoff when he plays action $x$ and facing the distribution $\mu$. Then, for any strategy $f$, player $t$ 's payoff function is

$$
U_{n}(t)(f)=\int_{X} V_{n}(t)\left(x, \nu_{n} \circ f^{-1}\right) \mathrm{d} f_{t}(x) .
$$

We let $\mathcal{H}_{m}$ denote this class of games.

Since $\mathcal{M}(X)$ is (homeomorphic to) a closed subset of $\mathcal{M}(\mathcal{M}(X))$, then each game $G_{n}$ in which mixed strategies are allowed induces a game $\tilde{G}_{n}$ in $\mathcal{H}$ simply by restricting players' payoff functions to $X \times \mathcal{M}(X)$. Furthermore, if $K \subseteq \mathcal{U}_{m}$ is equicontinuous, then $\tilde{K} \subseteq \mathcal{U}$ defined by restricting the domain in this way is also equicontinuous. Thus, for all $K \subseteq \mathcal{U}_{m}$ and all $\varepsilon>0$, if $n$ is sufficiently large, then $\tilde{G}_{n}$ has a pure strategy $\varepsilon / 3$ - equilibrium $f: T_{n} \rightarrow X$. It is easy to see that $f$ is also an $\varepsilon$ - equilibrium of $G_{n}$ since for every $t \in T_{n}$ and $\tilde{f}_{t} \in \mathcal{M}(X)$,

$$
\begin{aligned}
V_{n}(t)\left(f(t), \nu_{n} \circ f^{-1}\right) & \geq \int_{X} V_{n}(t)\left(x, \nu_{n} \circ f^{-1}\right) \mathrm{d} \tilde{f}_{t}(x)-\frac{2 \varepsilon}{3} \\
& \geq \int_{X} V_{n}(t)\left(x, \nu_{n} \circ\left(f \backslash_{t} \tilde{f}_{t}\right)^{-1}\right) \mathrm{d} \tilde{f}_{t}(x)-\varepsilon .
\end{aligned}
$$

Note that both inequalities hold since $V_{n}\left(T_{n}\right)$ is a subset of an equicontinuous family in $\mathcal{U}_{m}$ and if $n$ is sufficiently large to imply that the change from $\nu_{n} \circ\left(f \backslash_{t} x\right)^{-1}$ to $\nu_{n} \circ f^{-1}$ and from $\nu_{n} \circ f^{-1}$ to $\nu_{n} \circ\left(f \backslash_{t} \tilde{f}_{t}\right)^{-1}$, respectively, produces at most a change of $\varepsilon / 3$ in players' payoffs. Thus, we obtain the following existence result.

Theorem 5 Let $K$ be an equicontinuous subset of $\mathcal{U}_{m}$. Then, for all $\varepsilon>0$ there exists $N \in \mathbb{N}$ such that $n \geq N$ and $V_{n}\left(T_{n}\right) \subseteq K$ imply that $G_{n} \in \mathcal{H}_{m}$ has a pure $\varepsilon$ - equilibrium. 
An interesting question that arises once we consider mixed strategy is if all Nash equilibria can be approximately purified. In Carmona (2005a), we show that the answer is no.

\section{$7 \quad$ On the Need for Equicontinuity}

In this section, we show that in general, we cannot obtain a pure strategy Nash equilibrium even in equicontinuous large games. The exact version of Theorem 2 (i.e., with $\varepsilon=0$ ) therefore holds only in the limit case of games with a continuum of players. As an easy consequence, we also show that it is not possible to dispense with the equicontinuity assumption used in Theorem 2 .

The idea of the example is simple: each player has two actions, zero or one, to choose from. Player 1 wants to mismatch the average (if everyone else chooses 1 , he prefers 0 , and vice versa), while player 2 wants to match. For half of the remaining players, 0 is the dominant strategy, while 1 is the dominant strategy for the other half. This last condition forces the average to close to $1 / 2$ and pins down the behavior of all the players, except for players 1 and 2. At this point, we are in a matching pennies situation: if a strategy prescribes different actions for them, then player 2 would like to deviate to match player 1's choice; if a strategy prescribes the same action for both of them, then player 1 would like to deviate to mismatch player 2's choice. Hence, no pure equilibrium can exist.

Here are the details. Let $n \geq 4$ be even. Let $T_{n}=\{1, \ldots, n\}$ be partitioned into four sets: $T_{n, 1}=\{1\}, T_{n, 2}=\{2\}, T_{n, 3}$ and $T_{n, 4}$ satisfying 
$\left|T_{n, 3}\right|=\left|T_{n, 4}\right|=n / 2-1$.

Let $X=\{0,1\}$. Let $i: X \rightarrow X$ be the identity function, $\nu_{n}$ be the uniform measure on $T_{n}$ and $\mu \in \mathcal{M}(X)$.

Let $\alpha_{n}=1$ (below, we will change it). Define payoff functions as follows:

$$
V_{n}(1)(x, \mu)=\alpha_{n}(-1)^{x}\left(\int_{X} i \mathrm{~d} \mu-\frac{1}{2}\right) .
$$

For player 2, define $V_{n}(2)(x, \mu)=-V_{n}(1)(x, \mu)$.

Define

$$
W_{3}(x, \mu)=\left\{\begin{array}{rll}
10 & \text { if } & x=1 \\
0 & \text { if } & x=0 .
\end{array}\right.
$$

and $W_{4}=-W_{3}$. For a player $t \in T_{3}$, let $V_{n}(t)=W_{3}$, while for a player $t \in T_{4}$ let $V_{n}(t)=W_{4}$.

Let $G_{n}$ be as defined above whenever $n \geq 4$ is even. In the other case, define $G_{n}$ by setting $V_{n}(t)=V_{3}$ for all $t \in T_{n}$.

Note that if $f: T_{n} \rightarrow X$ is a strategy then

$$
\int_{X} i \mathrm{~d} \nu_{n} \circ f^{-1}=\sum_{t=1}^{n} \frac{f(t)}{n} .
$$

We claim that if $n \geq 4$ is even, then $G_{n}$ has no pure strategy Nash equilibrium. We prove the above claim by contradiction. Let $f$ be a Nash equilibrium. Then,

$$
f(t)=\left\{\begin{array}{lll}
1 & \text { if } & t \in T_{3} \\
0 & \text { if } & t \in T_{4}
\end{array}\right.
$$

Regarding $f(1)$ and $f(2)$, we consider the four possible cases.

Case 1 is when $f(1)=1$ and $f(2)=0$. In this case,

$$
\sum_{t=1}^{n} \frac{f(t)}{n}=\frac{1}{2}
$$


and

$$
\sum_{t=1}^{n} \frac{\left(f \backslash_{2} 1\right)(t)}{n}=\frac{1}{2}+\frac{1}{n} .
$$

Therefore, $V_{n}(2)\left(f(2), \nu_{n} \circ f^{-1}\right)=0$ and $V_{n}(2)\left(1, \nu_{n} \circ\left(f \backslash_{2} 1\right)^{-1}\right)=\alpha_{n}\left(\frac{1}{2}+\frac{1}{n}-\frac{1}{2}\right)$. Hence,

$$
V_{n}(2)\left(1, \nu_{n} \circ\left(f \backslash_{2} 1\right)^{-1}\right)-V_{n}(2)\left(f(2), \nu_{n} \circ f^{-1}\right)=\frac{\alpha_{n}}{n}=\frac{1}{n}>0,
$$

a contradiction.

Case 2 is when $f(1)=0$ and $f(2)=1$. Then, $\sum_{t=1}^{n} \frac{f(t)}{n}=\frac{1}{2}$ and $\sum_{t=1}^{n} \frac{\left(f \backslash_{2} 0\right)(t)}{n}=\frac{1}{2}-\frac{1}{n}$. Therefore, $V_{n}(2)\left(0, \nu_{n} \circ\left(f \backslash_{2} 0\right)^{-1}\right)-V_{n}(2)\left(f(2), \nu_{n} \circ\right.$ $\left.f^{-1}\right)=\frac{\alpha_{n}}{n}=\frac{1}{n}>0$, a contradiction.

Case 3 is when $f(1)=f(2)=0$. In this case, $\sum_{t=1}^{n} \frac{f(t)}{n}=\frac{n / 2-1}{n}=\frac{1}{2}-\frac{1}{n}$ and $\sum_{t=1}^{n} \frac{\left(f \backslash_{1} 1\right)(t)}{n}=\frac{1}{2}$. Therefore, $V_{n}(1)\left(1, \nu_{n} \circ\left(f \backslash_{1} 1\right)^{-1}\right)-V_{n}(1)\left(f(1), \nu_{n} \circ\right.$ $\left.f^{-1}\right)=\frac{\alpha_{n}}{n}=\frac{1}{n}>0$, a contradiction.

Finally, we consider the case $f(1)=f(2)=1$. In this case, $\sum_{t=1}^{n} \frac{f(t)}{n}=$ $\frac{n / 2-1+2}{n}=\frac{1}{2}+\frac{1}{n}$ and $\sum_{t=1}^{n} \frac{\left(f \backslash_{1} 0\right)(t)}{n}=\frac{1}{2}$. Therefore, $V_{n}(1)\left(0, \nu_{n} \circ\left(f \backslash_{1} 0\right)^{-1}\right)-$ $V_{n}(1)\left(f(1), \nu_{n} \circ f^{-1}\right)=\frac{\alpha_{n}}{n}=\frac{1}{n}>0$, a contradiction. This proves the claim.

Note that the family of payoff functions used, which equals

$$
\left\{V_{1}(1), V_{1}(2), W_{3}, W_{4}\right\}
$$

is equicontinuous. But, despite this, we cannot guarantee the existence of a pure strategy Nash equilibrium, even if $n$ is large.

This example can be easily modified to show that the conclusion of Theorem 2 fails, if we drop the equicontinuity assumption. Simply define $\alpha_{n}=2 n$, for all $n \in \mathbb{N}$. Similarly to what we have done above, we can show that, if 
$n \geq 4$ is even, then $G_{n}$ has no pure 1 - equilibrium. ${ }^{2}$

Note that, in this case, the family of payoff functions is equal to

$$
\left\{V_{3}, V_{4}\right\} \bigcup \cup_{n=1}^{\infty}\left\{V_{n}(1), V_{n}(2)\right\}
$$

Since $\lim _{n \rightarrow \infty} \alpha_{n}=\infty$, it follows that it is not equicontinuous. This accounts for the failure of the conclusion of Theorem 2 .

\section{Relation with Khan and Sun (1999)}

Khan and Sun (1999) stated an asymptotic existence theorem for large games based on non-standard methods. For the class of games we consider here, they state the following result: Let $\left\{G_{n}\right\}_{n=1}^{\infty} \subseteq \mathcal{H}$ be a tight sequence of games (i.e., the sequence $\left\{\nu_{n} \circ V_{n}^{-1}\right\}_{n=1}^{\infty} \subseteq \mathcal{M}(\mathcal{U})$ is tight). Then, for all $\varepsilon>0$ there exists $N \in \mathbb{N}$ such that for all $n \geq N$ there exists $f: T_{n} \rightarrow X$ such that for all $t \in T_{n}$ and all $x \in X$,

$$
V_{n}(t)\left(f(t), \nu_{n} \circ f^{-1}\right) \geq V_{n}(t)\left(x, \nu_{n} \circ f^{-1}\right)-\varepsilon .
$$

However, we can modify the example presented in Section 7 to show that this statement is false. ${ }^{3}$

\footnotetext{
${ }^{2}$ Again, we assume that a 1 - equilibrium exists. Then, players in $T_{3}$ must play 1 and players in $T_{4}$ must play 0 . We then consider the four possible case for players 1 and 2 . The above formulas show that there is at least one player whose gain from deviation is $\alpha_{n} / n=2>1$, which is a contradiction.

${ }^{3}$ The reason we need to modify the example is that our equilibrium notion is different than theirs. Theirs assumes that a player's deviation does not affect the distribution of actions. Note that the family of payoff functions used in our second example in Section 7 (i.e., when $\alpha_{n}=2 n$ ) is tight, and so it also shows that the above conclusion fails for our equilibrium concept.
} 
The difficulty with the statement in Khan and Sun (1999) is that there is a small fraction of players whose payoff function might be extremely sensitive to small changes in the distribution of choices. Thus, we cannot guarantee that those players are almost optimizing.

This suggests that their result is valid if we define approximate equilibria by requiring that a large fraction of players is almost optimizing. Formally, for all $\varepsilon, \eta \geq 0$, a strategy $f$ is an $(\varepsilon, \eta)$ - equilibrium of $G_{n}$ if

$$
\frac{\mid\left\{t \in T_{n}: U_{n}(t)(f) \geq U_{n}(t)\left(f \backslash_{t} x\right)-\varepsilon \text { for all } x \in X\right\} \mid}{n} \geq 1-\eta .
$$

Regarding this notion, we obtain the following existence result. ${ }^{4}$

Theorem 6 Let $\Gamma \subseteq \mathcal{H}$ be a tight family of games. Then, for all $\varepsilon>0$ and $\eta>0$ there exists $N \in \mathbb{N}$ such that $n \geq N$ and implies that $G_{n} \in \Gamma$ has a pure $(\varepsilon, \eta)$ - equilibrium.

This is the correct statement of Kahn and Sun's result, which was also pointed out by Sun (2005). This result is, however, equivalent to our Theorem $2 .^{5}$

Theorem 7 Theorem 2 holds if and only if Theorem 6 holds.

Of course, this implies that Kahn and Sun's theorem is also equivalent to Mas-Colell's. In fact, this is how we prove the above result: it is easier to compare both our Theorem 2 and Kahn and Sun's with Mas-Colell's than to compare them directly. As is typically the case, non-atomic games are easier to study and are, therefore, useful even when one is only interested in large finite games.

\footnotetext{
${ }^{4}$ Its proof is a simple variation of that of Theorem 2 and is presented in Appendix A.4.

${ }^{5}$ Its proof is a simple variation of that of Theorem 4 and is presented in Appendix A.5.
} 


\section{Concluding Remarks}

The main objective of this paper is to argue for Mas-Colell's formalization of the equilibrium theory of large games in terms of distributions. We follow his approach and show that:

1. for any equicontinuous family of payoff functions and any $\varepsilon>0$, all sufficiently large games have a pure $\varepsilon$ - equilibrium;

2. the above asymptotic result is equivalent to Mas-Colell's and

3. it is also equivalent to Khan and Sun's.

Therefore, we can construct an equilibrium theory of large finite games using Mas-Colell's model, which is as rich as the one developed by Kahn and Sun (and as rich as the theory obtained by Mas-Colell for games with a continuum of players). To put it differently, the existence of pure strategy Nash equilibria in games with a continuum of players can be studied equivalently to the existence of pure approximate equilibria in large, equicontinuous games.

These results provide an additional example of the type of results showing that approximate equilibria of finite games have the same, or approximately the same, properties as equilibria of continuum games. In this way, it stresses the close relationship between equilibria of games with a continuum of players and approximate equilibria of games with a finite number of players. 


\section{A Appendix}

\section{A.1 Lemmata}

In this appendix, we prove several results needed for our main results. Lemma 1 deals with measures with a finite support, which can be thought of as a vector in some Euclidean space. Roughly, Lemma 1 says that the Prohorov distance between two measures whose support is contained in some finite set is proportional to their Euclidean distance.

Lemma 1 Let $\tau, \mu \in \mathcal{M}(X)$ be such that $\operatorname{supp}(\tau) \cup \operatorname{supp}(\mu) \subseteq \Psi$, where $\Psi$ is a finite set. If there exists $\varepsilon>0$ such that $\left|\tau_{l}-\mu_{l}\right| \leq \varepsilon$ for all $1 \leq l \leq|\Psi|$, then $\rho(\tau, \mu) \leq|\Psi| \varepsilon$.

Proof. Let $\varepsilon>0$ and $B \subseteq X$ be Borel measurable. Then,

$$
\begin{aligned}
\tau(B)= & \sum_{l \in \Psi \cap B} \tau_{l} \leq \sum_{l \in \Psi \cap B}\left(\mu_{l}+\varepsilon\right) \leq \\
& \sum_{l \in \Psi \cap B} \mu_{l}+|\Psi| \varepsilon \leq \\
& \mu\left(\bar{B}_{|\Psi| \varepsilon}(B)\right)+|\Psi| \varepsilon .
\end{aligned}
$$

Similarly, we can show that $\mu(B) \leq \tau\left(\bar{B}_{|\Psi| \varepsilon}(B)\right)+|\Psi| \varepsilon$. This implies that $\rho(\tau, \mu) \leq|\Psi| \varepsilon$.

Lemma 2 shows that in large games, deviations by a small fraction of players have a small impact on the distribution of actions.

Lemma 2 Let $G_{n} \in \mathcal{H}$ be a game and let $f$ and $g$ be strategies. If

$$
\frac{\left|\left\{t \in T_{n}: f(t) \neq g(t)\right\}\right|}{n} \leq \gamma,
$$


then

$$
\rho\left(\nu_{n} \circ f^{-1}, \nu_{n} \circ g^{-1}\right) \leq \gamma
$$

Proof. Let $\mu=\nu_{n} \circ f^{-1}$ and $\tau=\nu_{n} \circ g^{-1}$. Let $B \subseteq X$ be Borel measurable. Then,

$$
\begin{aligned}
& \tau(B)=\frac{|\{t: g(t) \in B\}|}{n} \leq \frac{|\{t: f(t) \in B\}|}{n}+\frac{|\{t: f(t) \neq g(t)\}|}{n}= \\
& \mu(B)+\frac{|\{t: f(t) \neq g(t)\}|}{n} \leq \\
& \mu\left(\bar{B}_{\gamma}(B)\right)+\gamma .
\end{aligned}
$$

Similarly, we can show that $\mu(B) \leq \tau\left(\bar{B}_{\gamma}(B)\right)+\gamma$. This implies that $\rho(\tau, \mu) \leq$ $\gamma$

In particular, we have that $\rho\left(\nu_{n} \circ f^{-1}, \nu_{n} \circ\left(f \backslash_{t} x\right)^{-1}\right) \leq 1 / n$ for all strategies $f$, players $t \in T_{n}$ and actions $x \in X$.

Lemma 3 establishes the existence of an approximate equilibrium distribution with finite support for games with finitely many characteristics.

Lemma 3 Suppose that $\mu \in \mathcal{M}(\mathcal{U})$ has finite support and let $\tau$ be an equilibrium distribution of $\mu$. Then, there exists a sequence $\left\{\tau_{k}\right\}_{k=1}^{\infty} \subseteq \mathcal{M}(\mathcal{U} \times X)$ such that

1. $\tau_{k}$ converges to $\tau$,

2. $\tau_{\mathcal{U}, k}=\mu$ for all $k$,

3. $\tau_{X, k}$ has finite support for all $k$,

4. $\operatorname{supp}\left(\tau_{k}\right) \subseteq \operatorname{supp}(\tau)$ for all $k$ and 
5. for all $\varepsilon>0$, there exists $K \in \mathbb{N}$ such that

$$
\tau_{k}\left(\left\{(u, x): u\left(x, \tau_{X, k}\right) \geq u\left(X, \tau_{X, k}\right)-\varepsilon\right\}\right)=1,
$$

for all $k \geq K$.

Proof. Let $\operatorname{supp}(\mu)=\left\{u_{1}, \ldots, u_{n}\right\}$. Letting $D_{i}=\left\{x \in X:\left(u_{i}, x\right) \in\right.$ $\operatorname{supp}(\tau)\}$ for all $1 \leq i \leq n$, the fact that $\tau_{\mathcal{U}}=\mu$ readily implies that $\operatorname{supp}(\tau)=\cup_{i=1}^{n}\left(\left\{u_{i}\right\} \times D_{i}\right)$. This follows because $\operatorname{supp}(\tau) \subseteq \cup_{i=1}^{n}\left(\left\{u_{i}\right\} \times X\right)$ since $\cup_{i=1}^{n}\left(\left\{u_{i}\right\} \times X\right)$ is closed and $\tau\left(\cup_{i=1}^{n}\left(\left\{u_{i}\right\} \times X\right)\right)=\sum_{i=1}^{n} \tau_{\mathcal{U}}\left(\left\{u_{i}\right\}\right)=1$.

For all $1 \leq i \leq n$, define $c_{i}=\mu\left(\left\{u_{i}\right\}\right)$ and

$$
\tau_{i}(B)=\tau\left(\left\{u_{i}\right\} \times B\right) / c_{i}
$$

for all Borel subsets $B$ of $X$. For latter use, we claim that

$$
\operatorname{supp}(\tau)=\cup_{i=1}^{n}\left(\left\{u_{i}\right\} \times \operatorname{supp}\left(\tau_{i}\right)\right)
$$

Since $\cup_{i=1}^{n}\left(\left\{u_{i}\right\} \times \operatorname{supp}\left(\tau_{i}\right)\right)$ is closed and

$$
\tau\left(\cup_{i=1}^{n}\left(\left\{u_{i}\right\} \times \operatorname{supp}\left(\tau_{i}\right)\right)\right)=\sum_{i} \tau\left(\left\{u_{i}\right\} \times \operatorname{supp}\left(\tau_{i}\right)\right)=\sum_{i} c_{i} \tau_{i}\left(\operatorname{supp}\left(\tau_{i}\right)\right)=1,
$$

it follows that $\operatorname{supp}(\tau) \subseteq \cup_{i=1}^{n}\left(\left\{u_{i}\right\} \times \operatorname{supp}\left(\tau_{i}\right)\right)$. Conversely, recall that $\operatorname{supp}(\tau)=\cup_{i=1}^{n}\left\{u_{i}\right\} \times D_{i}$. Since $\operatorname{supp}(\tau)$ is closed, one easily sees that $D_{i}$ is closed. Furthermore, $\sum_{i=1}^{n} \tau\left(\left\{u_{i}\right\} \times D_{i}\right)=\tau(\operatorname{supp}(\tau))=1$ and $\tau\left(\left\{u_{i}\right\} \times D_{i}\right) \leq$ $\tau\left(\left\{u_{i}\right\} \times X\right)=c_{i}$ imply that $\tau\left(\left\{u_{i}\right\} \times D_{i}\right)=c_{i}$, that is, $\tau_{i}\left(D_{i}\right)=1$. Therefore, $\operatorname{supp}\left(\tau_{i}\right) \subseteq D_{i}$ and so, $\left\{u_{i}\right\} \times \operatorname{supp}\left(\tau_{i}\right) \subseteq\left\{u_{i}\right\} \times D_{i}$. Finally, one obtains that $\cup_{i=1}^{n}\left(\left\{u_{i}\right\} \times \operatorname{supp}\left(\tau_{i}\right)\right) \subseteq \cup_{i=1}^{n}\left(\left\{u_{i}\right\} \times D_{i}\right)=\operatorname{supp}(\tau)$.

The measure $\tau_{i}$ can be thought of as a measure in $\operatorname{supp}\left(\tau_{i}\right)$, a closed (and therefore, compact) subset of $X$. Then, there exists a sequence $\left\{\tau_{i, k}\right\}_{k}$ 
such that $\operatorname{supp}\left(\tau_{i, k}\right)$ is a finite subset of $\operatorname{supp}\left(\tau_{i}\right)$ and $\tau_{i, k}$ converges to $\tau_{i}$ in $\operatorname{supp}\left(\tau_{i}\right)$. Since $\operatorname{supp}\left(\tau_{i}\right)$ is closed in $X$, then, by Lemma 6 , we have that $\tau_{i, k}$ converges to $\tau_{i}$ in $X$.

Let $C$ be a Borel measurable subset of $\mathcal{U} \times X$. Then, let $B_{i}=\{x \in X$ : $\left.\left(u_{i}, x\right) \in C\right\}$ and define

$$
\tau_{k}(C)=\sum_{i=1}^{n} c_{i} \tau_{i, k}\left(B_{i}\right) .
$$

Note that $\tau_{\mathcal{U}, k}\left(\left\{u_{i}\right\}\right)=c_{i}$ for all $1 \leq i \leq n$ and so $\tau_{\mathcal{U}, k}=\mu$ for all $k$. Also, it is clear that $\tau_{X, k}$ has finite support for all $k$. Furthermore, since $\tau_{k}\left(\left\{\left(u_{i}, x\right)\right\}\right)=c_{i} \tau_{i, k}(\{x\})$ and so $\tau_{k}\left(\left\{\left(u_{i}, x\right)\right\}\right)>0$ if and only if $\tau_{i, k}(\{x\})>0$, it follows that

$$
\operatorname{supp}\left(\tau_{k}\right)=\cup_{i=1}^{n}\left(\left\{u_{i}\right\} \times \operatorname{supp}\left(\tau_{i, k}\right)\right) .
$$

This immediatly implies that $\operatorname{supp}\left(\tau_{k}\right) \subseteq \operatorname{supp}(\tau)$ for all $k, \operatorname{since} \operatorname{supp}\left(\tau_{i, k}\right) \subseteq$ $\operatorname{supp}\left(\tau_{i}\right)$.

We claim that $\tau_{k}$ converges to $\tau$. This follows since, if $h: \mathcal{U} \times X \rightarrow \mathbb{R}$ is continuous and bounded, then $x \mapsto h\left(u_{i}, x\right)$ is also continuous and bounded for all $1 \leq i \leq n$, and so

$$
\begin{aligned}
& \int_{\mathcal{U} \times X} h \mathrm{~d} \tau_{k}=\sum_{i=1}^{n} \int_{\left\{u_{i}\right\} \times X} h \mathrm{~d} \tau_{k}=\sum_{i=1}^{n} \int_{X} h\left(u_{i}, x\right) \mathrm{d} \tau_{i, k}(x) \\
& \rightarrow \sum_{i=1}^{n} \int_{X} h\left(u_{i}, x\right) \mathrm{d} \tau_{i}(x)=\int_{\mathcal{U} \times X} h \mathrm{~d} \tau .
\end{aligned}
$$

We finally establish property 5. For convenience, for all $\eta \geq 0$ and $\sigma \in$ $\mathcal{M}(\mathcal{U} \times X)$, let $B_{\sigma}^{\eta}=\left\{(u, x) \in \mathcal{U} \times X: u\left(x, \sigma_{X}\right) \geq u\left(X, \sigma_{X}\right)-\eta\right\}$, and $B_{\sigma}=B_{\sigma}^{0}$ if $\eta=0$. Let $\varepsilon>0$. Since $B_{\tau}$ is closed and $\tau\left(B_{\tau}\right)=1$, then $\operatorname{supp}(\tau) \subseteq B_{\tau}$. Hence, $\operatorname{supp}\left(\tau_{k}\right) \subseteq B_{\tau}$ for all $k$. 
Let $\delta>0$ be such that $\max \{d(x, y), \rho(\mu, \nu)\}<\delta$ implies that $\mid u(x, \mu)-$ $u(y, \nu) \mid<\varepsilon / 2$ for all $u \in\left\{u_{1}, \ldots, u_{n}\right\}$.

Let $K \in \mathbb{N}$ be such that $\rho\left(\tau_{X, k}, \tau_{X}\right)<\delta$ for all $k \geq K$. Then, $\operatorname{supp}\left(\tau_{k}\right) \cap$ $B_{\tau} \subseteq \operatorname{supp}\left(\tau_{k}\right) \cap B_{\tau_{k}}^{\varepsilon}$, since if $(u, x) \in \operatorname{supp}\left(\tau_{k}\right) \cap B_{\tau}$ and $y \in X$ then $u\left(x, \tau_{X, k}\right)>u\left(x, \tau_{X}\right)-\varepsilon / 2 \geq u\left(y, \tau_{X}\right)-\varepsilon / 2>u\left(y, \tau_{X, k}\right)-\varepsilon$ since $u \in$ $\left\{u_{1}, \ldots, u_{n}\right\}$. So

$$
\tau_{k}\left(B_{\tau_{k}}^{\varepsilon}\right)=\tau_{k}\left(\operatorname{supp}\left(\tau_{k}\right) \cap B_{\tau_{k}}^{\varepsilon}\right) \geq \tau_{k}\left(\operatorname{supp}\left(\tau_{k}\right) \cap B_{\tau}\right)=\tau_{k}\left(\operatorname{supp}\left(\tau_{k}\right)\right)=1 .
$$

This concludes the proof.

Lemma 4 strengthens the conclusion of Lemma 3, whenever the set of players' characteristics is a finite subset of an equicontinuous family. It guarantees the existence of a finite set of actions with the property that all the above games have an approximate equilibrium strategy taking values in this finite set. The strength of this result is that the finite set works uniformly for all such games, i.e., it depends only on the equicontinuous set and on the degree of approximation desired.

Lemma 4 Let $K$ be an equicontinuous subset of $\mathcal{U}$. Then, for all $\eta>0$, there exists $\left\{x_{1}, \ldots, x_{m}\right\} \subseteq X$ such that the following holds:

1. $X=\cup_{i=1}^{m} B_{\eta}\left(x_{i}\right)$ and

2. if $G=(([0,1], \lambda), V, X)$ is such that $V([0,1])$ is finite, then there exists a strategy $g$ such that $g([0,1]) \subseteq\left\{x_{1}, \ldots, x_{m}\right\}$ and

$$
V(t)\left(g(t), \lambda \circ g^{-1}\right)>V(t)\left(X, \lambda \circ g^{-1}\right)-\eta
$$

for all $t \in V^{-1}(K)$. 
Consequently, if $V([0,1])$ is a subset of $K$, then $g$ is an $\eta$-equilibrium.

Proof. Let $\eta>0$. Since $K$ is equicontinuous, there exists $\delta>0$ such that $\max \{d(x, y), \rho(\mu, \nu)\}<\delta$ implies that $|u(x, \mu)-u(y, \nu)|<\eta / 3$ for all $x, y \in X, \mu, \nu \in \mathcal{M}(X)$ and $u \in K$. We can choose $\delta<\eta$.

Let $\left\{x_{1}, \ldots, x_{m}\right\}$ be such that $X=\cup_{j=1}^{m} B_{\delta / 2}\left(x_{j}\right)$. Define $B_{1}=B_{\delta / 2}\left(x_{1}\right)$ and $B_{j}=B_{\delta / 2}\left(x_{j}\right) \backslash\left(\cup_{l=1}^{j} B_{\delta / 2}\left(x_{l}\right)\right), 2 \leq j \leq m$.

Let $G$ be such that $V([0,1])$ is a finite subset of $\mathcal{U}$. By Mas-Colell Theorem, $G$ has an equilibrium distribution. It follows from Lemma 3 that $G$ has an $\eta / 3$ - equilibrium distribution with finite support and so a pure strategy $\eta / 3$ - equilibrium $f$ (this follows easily from Liaponov's Theorem (see Rudin (1971, Theorem 5.5, p. 120))).

Define $g:[0,1] \rightarrow\left\{x_{1}, \ldots, x_{m}\right\}$ by $g(t)=x_{j}$ if $f(t) \in B_{j}$. Then, clearly $|f(t)-g(t)|<\delta / 2$. This implies that

$$
\{t \in[0,1]: g(t) \in D\} \subseteq\left\{t \in[0,1]: f(t) \in \bar{B}_{\delta / 2}(D)\right\}
$$

for all Borel measurable subsets $D$ of $X$ and so

$$
\lambda \circ g^{-1}(D) \leq \lambda \circ f^{-1}\left(\bar{B}_{\delta / 2}(D)\right)+\delta / 2
$$

Similarly, one can show that $\lambda \circ f^{-1}(D) \leq \lambda \circ g^{-1}\left(\bar{B}_{\delta / 2}(D)\right)+\delta / 2$. Thus,

$$
\rho\left(\lambda \circ g^{-1}, \lambda \circ f^{-1}\right) \leq \delta / 2
$$

This implies that for all $t \in V^{-1}(K)$ and all $x \in X$,

$$
\begin{aligned}
V(t)\left(g(t), \lambda \circ g^{-1}\right) & >V(t)\left(f(t), \lambda \circ f^{-1}\right)-\frac{\eta}{3} \\
& \geq V(t)\left(x, \lambda \circ f^{-1}\right)-\frac{2 \eta}{3} \\
& >V(t)\left(x, \lambda \circ g^{-1}\right)-\eta .
\end{aligned}
$$


In particular, if $V([0,1]) \subseteq K$, then $g$ is an $\eta$ - equilibrium of $G$.

Lemma 5 is used to draw conclusions for games with a continuum of players from properties of large finite games. It uses the definition of $(\varepsilon, \eta)$ - equilibrium, given in Section 8. Furthermore, it considers a more general case in which a game $G_{n}=\left(\left(T_{n}, \nu_{n}\right), V_{n}, X\right)$ with finitely many players has $\left|T_{n}\right|$ players (not necessarily equal to $n$ ), and $\nu_{n}$ is the uniform measure on $T_{n}$.

Lemma 5 Let $G=(([0,1], \lambda), V, X)$ be a game with a continuum of players, $\tau$ be a distribution on $\mathcal{U} \times X$ satisfying $\tau_{\mathcal{U}}=\lambda \circ V^{-1}$ and $\varepsilon \geq 0$. Suppose that $\left\{G_{n}\right\}_{n=1}^{\infty}$ is a sequence of games with a finite number of players and $\left\{f_{n}\right\}_{n=1}^{\infty}$ is a sequence of strategies satisfying:

1. $\left|T_{n}\right| \rightarrow \infty$,

2. $f_{n}$ is an $\left(\varepsilon_{n}, \eta_{n}\right)$ - equilibrium of $G_{n}$,

3. $\varepsilon_{n} \geq \varepsilon, \varepsilon_{n} \rightarrow \varepsilon$,

4. $\eta_{n} \geq 0, \eta_{n} \rightarrow 0$ and

5. $\nu_{n} \circ\left(V_{n}, f_{n}\right)^{-1}$ converges to $\tau$,

then $\tau$ is an $\varepsilon$ - equilibrium distribution of $G$.

Lemma 5 is slightly more general than the sufficiency part of Theorem 1 in Carmona (2004). We include its proof for the sake of completeness.

Proof. Let $\tau_{n}=\nu_{n} \circ\left(V_{n}, f_{n}\right)^{-1}$. For all $x \in \operatorname{supp}\left(\tau_{n, X}\right)$ and $y \in X$, define $\tau_{n, X}^{x, y}$ as follows: $\tau_{n, X}^{x, y}(x)=\tau_{n, X}(x)-1 /\left|T_{n}\right|, \tau_{n, X}^{x, y}(y)=\tau_{n, X}(y)+1 /\left|T_{n}\right|$ and $\tau_{n, X}^{x, y}(\bar{x})=\tau_{n, X}(\bar{x})$ for all $\bar{x}$ in the support of $\tau_{n, X}$ that are different from $x$ and 
from $y$. Let $B_{\tau_{n}}^{\varepsilon_{n}}=\left\{(u, x) \in \operatorname{supp}\left(\tau_{n}\right): u\left(x, \tau_{n, X}\right) \geq u\left(y, \tau_{n, X}^{x, y}\right)-\varepsilon_{n}\right.$ for all $y \in$ $X\}$. Also, let $B_{\tau}^{\varepsilon}=\left\{(u, x) \in \mathcal{U} \times X: u\left(x, \tau_{X}\right) \geq u\left(X, \tau_{X}\right)-\varepsilon\right\}$.

Since $\tau_{n}$ converges to $\tau$, then $\tau_{X, n}$ converges to $\tau_{X}$. Taking a subsequence if necessary, we can assume that $\left|T_{n}\right| \nearrow \infty, \rho\left(\tau_{X}, \tau_{X, n}\right) \searrow 0, \varepsilon_{n} \searrow \varepsilon$ and $\eta_{n} \searrow 0$. Let $\theta_{n}=\rho\left(\tau_{X}, \tau_{X, n}\right)+2 /\left|T_{n}\right|$; clearly, $\theta_{n} \searrow 0$. Since, for all $x \in \operatorname{supp}\left(\tau_{n, X}\right)$ and $y \in X$,

$$
\begin{aligned}
\rho\left(\tau_{X}, \tau_{n, X}^{x, y}\right) & \leq \rho\left(\tau_{X}, \tau_{X, n}\right)+\rho\left(\tau_{n, X}, \tau_{n, X}^{x, y}\right) \\
& \leq \rho\left(\tau_{X}, \tau_{X, n}\right)+\frac{1}{\left|T_{n}\right|},
\end{aligned}
$$

it follows that $\rho\left(\tau_{X}, \tau_{n, X}^{x, y}\right)<\theta_{n}$.

Define, for each $u \in \mathcal{U}$,

$$
\beta_{n}(u)=\sup _{x \in X, \nu \in \mathcal{M}(X)}\left\{\left|u(x, \nu)-u\left(x, \tau_{X}\right)\right|: \rho\left(\nu, \tau_{X}\right)<\theta_{n}\right\} .
$$

Since $u$ is continuous on $X \times \mathcal{M}(X)$, which is compact, it follows that $u$ is uniformly continuous. Thus, $\beta_{n}(u) \searrow 0$ as $n \rightarrow \infty$. We claim that $\beta_{n}$ is continuous in $\mathcal{U}$.

Let $\eta>0$. Define $\delta<\eta / 2$. Then if $\|u-v\|=\sup _{y \in X, \phi \in \mathcal{M}(X)} \mid u(y, \phi)-$ $v(y, \phi) \mid<\delta$, we have for any $x \in X$, and $\nu \in \mathcal{M}(X)$ such that $\rho\left(\nu, \tau_{X}\right)<\theta_{n}$

$$
\begin{aligned}
\left|v(x, \nu)-v\left(x, \tau_{X}\right)\right| & \leq|v(x, \nu)-u(x, \nu)|+\left|u(x, \nu)+u\left(x, \tau_{X}\right)\right|+ \\
& +\left|v\left(x, \tau_{X}\right)-u\left(x, \tau_{X}\right)\right|<\delta+\beta_{n}(u)+\delta
\end{aligned}
$$

and so $\beta_{n}(v) \leq 2 \delta+\beta_{n}(u)<\eta+\beta_{n}(u)$. By symmetry, $\beta_{n}(u)<\eta+\beta_{n}(v)$, and so $\left|\beta_{n}(u)-\beta_{n}(v)\right|<\eta$. Hence, $\beta_{n}$ is continuous, as claimed.

Given the definition of $\beta_{n}$, we have that $B_{\tau_{n}}^{\varepsilon_{n}} \subseteq D_{n}:=\{(u, x) \in \mathcal{U} \times X$ : $\left.u\left(x, \tau_{X}\right) \geq u\left(X, \tau_{X}\right)-\varepsilon_{n}-2 \beta_{n}(u)\right\}$. Since $\beta_{n}$ is continuous, we see that $D_{n}$ is closed, and so Borel measurable. Thus, $\tau_{n}\left(D_{n}\right) \geq 1-\eta_{n}$. Also, $D_{n} \searrow B_{\tau}^{\varepsilon}$. 
Let $n \in \mathbb{N}$ be given. Then, if $k \geq n$, it follows that $\tau_{k}\left(D_{n}\right) \geq \tau_{k}\left(D_{k}\right) \geq 1-$ $\eta_{k} \geq 1-\eta_{n}$, and so $\tau\left(D_{n}\right) \geq \lim \sup _{j} \tau_{j}\left(D_{n}\right) \geq 1-\eta_{n}$ by Parthasarathy (1967, II.6.1(c)). Hence, $\tau\left(B_{\tau}^{\varepsilon}\right)=\lim _{n} \tau\left(D_{n}\right)=1$. Therefore, $\tau$ is an equilibrium distribution of $G$.

The following lemma shows that the convergence of a sequence of measures can be studied with respect to any closed set containing both their support and the support of the limit measure.

Lemma 6 Let $X$ be a metric space and $\mu, \mu_{n} \in \mathcal{M}(X)$ for all $n \in \mathbb{N}$. Let $C$ be a closed subset of $X$ satisfying $\operatorname{supp}\left(\mu_{n}\right), \operatorname{supp}(\mu) \subseteq C$. Then, $\mu_{n} \Rightarrow \mu$ in $C$ if and only if $\mu_{n} \Rightarrow \mu$ in $X$.

Proof. (Necessity) Let $h: X \rightarrow \mathbb{R}$ be bounded and continuous. Then $h_{\mid C}$ is also bounded and continuous, and so

$$
\int_{C} h \mathrm{~d} \mu_{n} \rightarrow \int_{C} h \mathrm{~d} \mu
$$

Thus,

$$
\int_{X} h \mathrm{~d} \mu_{n}=\int_{C} h \mathrm{~d} \mu_{n} \rightarrow \int_{C} h \mathrm{~d} \mu=\int_{X} h \mathrm{~d} \mu .
$$

It follows that $\mu_{n} \Rightarrow \mu$ in $X$.

(Sufficiency) Let $h: C \rightarrow \mathbb{R}$ be bounded and continuous. Then by the Tietze-Urysohn extension theorem, there exists a bounded, continuous function $h^{*}: X \rightarrow \mathbb{R}$ such that $h^{*}(x)=h(x)$ for all $x \in C$. Therefore,

$$
\int_{X} h^{*} \mathrm{~d} \mu_{n} \rightarrow \int_{X} h^{*} \mathrm{~d} \mu
$$

and

$$
\int_{X} h^{*} \mathrm{~d} \mu=\int_{C} h^{*} \mathrm{~d} \mu=\int_{C} h \mathrm{~d} \mu
$$


(and similarly, $\int_{X} h^{*} \mathrm{~d} \mu_{n}=\int_{C} h \mathrm{~d} \mu_{n}$ for all $n \in \mathbb{N}$ ). Therefore, $\int_{C} h \mathrm{~d} \mu_{n} \rightarrow$ $\int_{C} h \mathrm{~d} \mu$. It follows that $\mu_{n} \Rightarrow \mu$ in $C$.

\section{A.2 Proof of Corollaries 1, 3 and 4}

The proof of each corollary follows the same idea. Hence, we will present it only for the case of Corollary 1.

Let $\varepsilon>0$. Let $V \in K$ and $i: X \rightarrow X$ denote the identity function in $X$. Define $v: X \times \mathcal{M}(X) \rightarrow \mathbb{R}$ by

$$
v(x, \mu)=V\left(x, \int_{X} i \mathrm{~d} \mu\right)
$$

for all $x \in X$ and $\mu \in \mathcal{M}(X)$.

Note that $i$ is Bochner integrable and $\int_{X} i \mathrm{~d} \mu \in \overline{\mathrm{co}}(X)$ for all $\mu \in \mathcal{M}(X)$. Furthermore, if $\mu_{k}$ converges to $\mu$, then $\int_{X} i \mathrm{~d} \mu_{k}$ converges weakly to $\int_{X} i \mathrm{~d} \mu$ and so $v$ is continuous.

Let $g: X \times \mathcal{M}(X) \rightarrow X \times \overline{\mathrm{co}}(X)$ be defined by $g(x, \mu)=\left(x, \int_{X} i \mathrm{~d} \mu\right)$. In order to apply Theorem 2, it remains to show that $\tilde{K}$ defined by $\{u \in \mathcal{U}$ : $u=v \circ g$ for some $v \in K\}$ is equicontinuous.

Let $d$ denote the metric in $\overline{\mathrm{co}}(X)$. Let $\varepsilon>0$ and let $\delta>0$ be such that $|v(x, z)-v(y, w)|<\varepsilon$ whenever $\max \{d(x, y), d(z, w)\}<\delta$ and $v \in K$.

Note that it is enough to show that there exists $\eta>0$ such that $\rho(\mu, \tau)<\eta$ implies $d\left(\int_{X} i \mathrm{~d} \mu, \int_{X} i \mathrm{~d} \tau\right)<\delta$.

Suppose, in order to reach a contradiction, that such $\eta>0$ does not exist. Then, for all $k \in \mathbb{N}$ there are $\left\{\mu_{k}\right\}$ and $\left\{\tau_{k}\right\}$ such that $\rho\left(\mu_{k}, \tau_{k}\right)<1 / k$ and $d\left(\int_{X} i \mathrm{~d} \mu_{k}, \int_{X} i \mathrm{~d} \tau_{k}\right) \geq \delta$. Since $\mathcal{M}(X)$ is compact, we may assume that there exists $\mu$ such that $\mu_{k}$ converges to $\mu$. Then, $\tau_{k}$ converges to $\mu$ and so both 
$\int_{X} i \mathrm{~d} \mu_{k}$ and $\int_{X} i \mathrm{~d} \tau_{k}$ converge weakly to $\int_{X} i \mathrm{~d} \mu$. Hence, $d\left(\int_{X} i \mathrm{~d} \mu_{k}, \int_{X} i \mathrm{~d} \tau_{k}\right) \rightarrow$ 0 , which is a contradiction.

Thus, $\tilde{K}$ is an equicontinuous subset of $\mathcal{U}$. By Theorem 2, let $N \in \mathbb{N}$ be such that $n \geq N$ implies that all games $\tilde{G}_{n} \in \mathcal{H}$ with $\tilde{V}\left(T_{n}\right) \subseteq \tilde{K}$ have an $\varepsilon$ - equilibrium.

Let $n \geq N$ and $G_{n} \in \mathcal{H}_{w}$ be such that $V\left(T_{n}\right) \subseteq K$. Define $\tilde{G}_{n} \in \mathcal{H}$ by letting $\tilde{V}_{t}=V_{t} \circ g$. Then, $\tilde{V}\left(T_{n}\right) \subseteq \tilde{K}$ and so there exists an $\varepsilon$ - equilibrium $f: T_{n} \rightarrow X$. Thus,

$$
\tilde{V}_{t}\left(f(t), \nu_{n} \circ f^{-1}\right) \geq \tilde{V}\left(x, \nu_{n} \circ\left(f \backslash_{t} x\right)^{-1}\right)-\varepsilon
$$

for all $t \in T_{n}$ and $x \in X$.

Note that

$$
\begin{gathered}
\tilde{V}_{t}\left(f(t), \nu_{n} \circ f^{-1}\right)=V_{t} \circ g\left(f(t), \nu_{n} \circ f^{-1}\right)=V_{t}\left(f(t), \int_{X} i \mathrm{~d} \nu_{n} \circ f^{-1}\right), \\
\tilde{V}_{t}\left(x, \nu_{n} \circ\left(f \backslash_{t} x\right)^{-1}\right)=V_{t}\left(x, \int_{X} i \mathrm{~d} \nu_{n} \circ\left(f \backslash_{t} x\right)^{-1}\right), \\
\int_{X} i \mathrm{~d} \nu_{n} \circ f^{-1}=\frac{1}{n} \sum_{t=1}^{n} f(t)=\int_{T_{n}} f \mathrm{~d} \nu_{n}
\end{gathered}
$$

and

$$
\int_{X} i \mathrm{~d} \nu_{n} \circ\left(f \backslash_{t} x\right)^{-1}=\frac{1}{n} \sum_{\tilde{t}=1}^{n}\left(f \backslash_{t} x\right)(\tilde{t})=\int_{T_{n}}\left(f \backslash_{t} x\right) \mathrm{d} \nu_{n} .
$$

Therefore,

$$
V_{t}\left(f(t), \int_{T_{n}} f \mathrm{~d} \nu_{n}\right) \geq V_{t}\left(x, \int_{T_{n}}\left(f \backslash_{t} x\right) \mathrm{d} \nu_{n}\right)-\varepsilon
$$

and so $f$ is an $\varepsilon$ - equilibrium of $G_{n}$. 


\section{A.3 Example for Section 8}

Let $n \geq 4$ be even. Let $T_{n}=\{1, \ldots, n\}$ be partitioned into four sets: $T_{n, 1}=$ $\{1\}, T_{n, 2}=\{2\}, T_{n, 3}$ and $T_{n, 4}$ satisfying $\left|T_{n, 3}\right|=\left|T_{n, 4}\right|=n / 2-1$.

Let $X=\{0,1\}$. Let $i: X \rightarrow X$ be the identity function, $\nu_{n}$ be the uniform measure on $T_{n}$ and $\mu \in \mathcal{M}(X)$.

Let $\gamma>1$,

$$
\frac{1}{1+2 / n}<\beta_{n}<1
$$

and

$$
\alpha_{n}=\max \left\{\frac{2}{\beta_{n}+2 \beta_{n} / n-1}, \frac{2}{1-\beta_{n}+2 \beta_{n} / n}, \frac{2}{1-\beta_{n}}\right\} .
$$

Clearly, $\alpha_{n}>0$. In fact, $\alpha_{n} \rightarrow \infty$ since $\beta_{n} \rightarrow 1$.

Define payoff functions as follows:

$$
V_{n}(1)(x, \mu)=\alpha_{n}(-1)^{x}\left(\beta_{n} \int_{X} i \mathrm{~d} \mu-\frac{1}{2}\right)
$$

For player 2, define

$$
W_{2}(x, \mu)=\frac{2}{\gamma-1}(-1)^{x}\left(\frac{1}{2}-\gamma \int_{X} i \mathrm{~d} \mu\right) .
$$

and $V_{n}(2)=W_{2}$.

Define

$$
W_{3}(x, \mu)=\left\{\begin{array}{rll}
10 & \text { if } & x=1 \\
0 & \text { if } & x=0
\end{array}\right.
$$

and $W_{4}=-W_{3}$. For a player $t \in T_{3}$, let $V_{n}(t)=W_{3}$, while for a player $t \in T_{4}$ let $V_{n}(t)=W_{4}$.

Let $G_{n}$ be as defined above whenever $n \geq 4$ is even. In the other case, define $G_{n}$ by setting $V_{n}(t)=W_{3}$ for all $t \in T_{n}$. Clearly, the sequence $\left\{G_{n}\right\}_{n=1}^{\infty}$ 
is tight: let $\varepsilon>0$ and let $N \in \mathbb{N}$ be such that $1 / N<\varepsilon$. Then, define

$$
K=\left\{W_{2}, W_{3}, W_{4}\right\} \bigcup \cup_{n=1}^{N}\left\{V_{n}(1)\right\}
$$

We have that $\nu_{n} \circ V_{n}^{-1}(K) \geq 1-1 / n>1-\varepsilon$ for all $n \in \mathbb{N}$.

We claim that if $n \geq 4$ is even, then there is no $f: T_{n} \rightarrow X$ such that, for all $t \in T_{n}$ and $x \in X$,

$$
V_{n}(t)\left(f(t), \nu_{n} \circ f^{-1}\right) \geq V_{n}(t)\left(x, \nu_{n} \circ f^{-1}\right)-1
$$

We prove the above claim by contradiction. Let $f$ be a 1 - equilibrium. Then, it follows that $f(t)=1$ for all $t \in T_{3}$ and $f(t)=0$ for all $t \in T_{4}$. Regarding $f(1)$ and $f(2)$, we consider the four possible cases.

Case 1 is when $f(1)=1$ and $f(2)=0$. In this case, $\sum_{t=1}^{n} \frac{f(t)}{n}=\frac{1}{2}$. Therefore, $V_{n}(2)\left(1, \nu_{n} \circ f^{-1}\right)-V_{n}(2)\left(f(2), \nu_{n} \circ f^{-1}\right)=\frac{2}{\gamma-1}(\gamma-1)=2>1$, a contradiction.

Case 2 is when $f(1)=0$ and $f(2)=1$. Then, $\sum_{t=1}^{n} \frac{f(t)}{n}=\frac{1}{2}$. Therefore, $V_{n}(1)\left(1, \nu_{n} \circ f^{-1}\right)-V_{n}(1)\left(f(1), \nu_{n} \circ f^{-1}\right)=\alpha_{n}\left(1-\beta_{n}\right) \geq 2>1$, a contradiction.

Case 3 is when $f(1)=f(2)=0$. In this case, $\sum_{t=1}^{n} \frac{f(t)}{n}=\frac{n / 2-1}{n}=\frac{1}{2}-\frac{1}{n}$. Therefore, $V_{n}(1)\left(1, \nu_{n} \circ f^{-1}\right)-V_{n}(1)\left(f(1), \nu_{n} \circ f^{-1}\right)=\alpha_{n}\left(1-\beta_{n}+2 \beta_{n} / n\right) \geq$ $2>1$, a contradiction.

Finally, we consider the case $f(1)=f(2)=1$. In this case, $\sum_{t=1}^{n} \frac{f(t)}{n}=$ $\frac{n / 2-1+2}{n}=\frac{1}{2}+\frac{1}{n}$. Therefore, $V_{n}(1)\left(0, \nu_{n} \circ f^{-1}\right)-V_{n}(1)\left(f(1), \nu_{n} \circ f^{-1}\right)=$ $\alpha_{n}\left(\beta_{n}+2 \beta_{n} / n-1\right) \geq 2>1$, a contradiction. This proves the claim.

Note that the family of payoff functions used, which equals

$$
\left\{W_{2}, W_{3}, W_{4}\right\} \bigcup \cup_{n=1}^{\infty}\left\{V_{n}(1)\right\}
$$

is not equicontinuous. This is due to the fact that $\lim _{n \rightarrow \infty} \alpha_{n}=\infty$. 


\section{A.4 Proof of Theorem 6}

Theorem 6 can be proven using the same scheme used to prove Theorem 2 . Let $\varepsilon>0$ and $\eta>0$. Let $K$ be such that $\nu_{n} \circ V_{n}^{-1}(K)>1-\eta$ for all $G_{n} \in \Gamma$. Then, let $\left\{x_{1}, \ldots, x_{m}\right\}$ be given by Lemma 4 . Following the proof of Theorem 2, we can show that

$$
V_{n}(t)\left(f(t), \nu_{n} \circ f^{-1}\right) \geq V_{n}(t)\left(x, \nu_{n} \circ\left(f \backslash_{t} x\right)^{-1}\right)-\varepsilon
$$

for all $x \in X$ and all $t \in V_{n}^{-1}(K)$. Since, $\nu_{n} \circ V_{n}^{-1}(K)>1-\eta$ for all $G_{n} \in \Gamma$, the result follows.

\section{A.5 Proof of Theorem 7}

It is enough to show that Theorem 6 implies Theorem 3. We can use the same argument used in the proof of Theorem 4, except that $f_{\gamma_{k} t_{k}}$ is only a $(1 / k, 1 / k)$ - equilibrium of $G_{\gamma_{k} t_{k}}$. However, Lemma 5 still applies and the conclusion follows.

\section{References}

Aumann, R. (1964): "Markets with a Continuum of Traders," Econometrica, 32, 39-50.

Carmona, G. (2004): "Nash Equilibria of Games with a Continuum of Players," Universidade Nova de Lisboa.

(2005a): "Large Games in Compact Metric Spaces: Purification of Nash Equilibria," Universidade Nova de Lisboa. 
(2005b): “On a Theorem by Mas-Colell," Universidade Nova de Lisboa.

Diestel, J., And J. J. Uhl (1977): Vector Measures. American Mathematical Society, Providence.

Dunford, N., And J. Schwartz (1957): Linear Operators Part I: General Theory. Wiley, New York.

Hildenbrand, W. (1974): Core and Equilibria of a Large Economy. Princeton University Press, Princeton.

Khan, M., K. Rath, and Y. Sun (1997): "On the Existence of Pure Strategy Equilibria in Games with a Continuum of Players," Journal of Economic Theory, 76, 13-46.

Khan, M., and Y. Sun (1995): "Pure Strategies in Games with Private Information," Journal of Mathematical Economics, 24, 633-653.

- (1999): "Non-Cooperative Games on Hyperfinite Loeb Spaces," Journal of Mathematical Economics, 31, 455-492.

Mas-Colell, A. (1984): "On a Theorem by Schmeidler," Journal of Mathematical Economics, 13, 201-206.

Nash, J. (1950): “Non-Cooperative Games," Ph.D. thesis, Princeton University.

Parthasarathy, K. (1967): Probability Measures on Metric Spaces. Academic Press, New York. 
RASHID, S. (1983): "Equilibrium Points of Non-atomic Games: Asymptotic Results," Economics Letters, 12, 7-10.

Rudin, W. (1971): Functional Analysis. McGraw-Hill, New York.

(1976): Principles of Mathematical Analysis. McGraw-Hill, New York

Schmeidler, D. (1973): "Equilibrium Points of Nonatomic Games," Journal of Statistical Physics, 4, 295-300.

Sun, Y. (2005): Private Communication. 